\title{
Advanced predictions of solidification in cryogenic natural gas and LNG processing
}

\author{
Corey Baker ${ }^{1}$, Arman Siahvashi' ${ }^{1}$ Jordan Oakley ${ }^{1}$, Thomas Hughes ${ }^{2}$, Darren Rowland ${ }^{1}$, \\ Stanley Huang ${ }^{3}$ and Eric F. May ${ }^{1}$
}

1. Fluid Science \& Resources Division, Department of Chemical Engineering, The University of Western Australia, 35 Stirling Hwy, Crawley 6009, AUSTRALIA

2. Oil \& Gas Engineering, Monash University, Clayton VIC, AUSTRALIA

3. Chevron Energy Technology Company, Houston TX 77002, USA

The formation and deposition of solids during the cryogenic processing of natural gas is a perennial risk for operators. Several tools are available for predicting the temperatures at which heavy hydrocarbon solids will form in cryogenic processing equipment; of these the Kohn and Luks Solids Solubility Program (KLSSP) from GPA Midstream has become an industry standard tool for predicting solid-fluid equilibria (SFE) in cryogenic processes. However, although it describes well many of the data sets generated as part of the GPA's research program in the 1970s and 1980s, the KLSSP suffers from limitations including fixed ranges of temperature, mixture composition, no dependence on pressure and a limited set of possible freeze-out components. Here, a new software tool called ThermoFAST is presented, which overcomes these limitations and has been endorsed by GPA Midstream to replace the KLSSP. This model uses a cubic equation of state and efficient flash algorithms to enable rapid calculations of solid-liquid, solid-vapor, and solid-liquid-vapor equilibrium conditions in addition to normal vapor-liquid phase envelopes. The model has been tuned to the available solid-fluid equilibrium literature data for 58 binary mixtures and is able to represent them with an average root-mean-squared temperature deviation of only $1.7 \mathrm{~K}$. It was thoroughly tested against available SLE data for multi-component LNG mixtures, and improved the accuracy of predicted melting temperatures by a factor of 7 relative to KLSSP, with an average rms deviation of only $2.0 \mathrm{~K}$. The rapid flash algorithm was used to identify 26 distinct transition pathways involving solidification phenomena in LNG-relevant fluids: this includes the prediction of retrograde solidification in multiple systems (including the methane + benzene binary) where a decrease in system temperature produces a reduction in the amount of solid phase present. 


\section{Introduction}

Natural gas accounts for nearly $22 \%$ of world energy production [1], with approximately $32 \%$ of global trade occurring through the production and transport of Liquefied Natural Gas (LNG) [2]. So-called 'mega-scale' production trains with capacities around (1 to 8) million tonnes per annum generate the vast majority of the LNG traded internationally, with volumes on the order of $200,000 \mathrm{~m}^{3}$ being shipped on a weekly basis from such plants [3-5]. The regularity of this shipment schedule is often central to the business model of mega-scale LNG production: decade-long supply contracts enable customers to satisfy their needs for energy security and diversity, while helping producers to secure the investment capital needed for plant construction and operation. Accordingly, the financial penalties associated with missing just one scheduled LNG shipment are significant given that, at the current spot price of US\$6 per GJ [6], the value of the LNG cargo is around US\$30 million. The severity of this consequence means that the risk associated with an unplanned shut-down of a mega-scale LNG plant is appreciable, and it is essential that the probability of technical problems capable of causing such an outcome be minimised.

The unwanted carry-over of heavy compounds into the vapour overheads that enter the MCHE represents a significant operational problem, particularly for mega-scale LNG plants. This can occur for several reasons including (1) variations in the feed entering the liquefaction plant, (2) the distillation column's operation is not adequate, (3) a process upset occurs upstream of the column or (4) the need to increase the heating value of the LNG through the re-injection of heavier hydrocarbons [7]. Carry-over of heavy compounds could lead to solid freeze-out within the MCHE. Deposition of solids reduces the heat transfer efficiency of the MCHE and leads to increased operational costs. Ultimately, a continued build-up of solids in the MCHE will result in a blockage and the unplanned shutdown of the plant. In a mega-scale LNG train where the consequences of such a shutdown are greatest, removal of the blockage and the subsequent plant re-start will require that the MCHE be taken through a $300 \mathrm{~K}$ temperature cycle. As the MCHE is both massive and sensitive to thermal shocks, any such temperature cycle is likely to take several days or a week. For this reason, an accurate knowledge of, and an ability to predict the solubility of impurities in LNG is highly important.

The foremost tool used in industry for predicting the solubility of hydrocarbon solids in cryogenic fluids is the Kohn and Luks Solids Solubility Program (KLSSP) from GPA 
Midstream [8]. This tool uses empirical functions based on activity coefficient models to reproduce closely certain experimental solubility data, especially those acquired during the extensive campaign headed by Kohn and Luks under the direction of GPA during the 1970s and '80s [9-23]. Unfortunately, several restrictions of KLSSP limit the viability of its future use. First, the empirical activity coefficient model employed in KLSSP contains no dependence on pressure. Second, the model inputs are narrowly restricted in terms of temperature (> $100 \mathrm{~K})$, available components and maximum composition of impurities, as shown in Table 1 . Third, the software can only determine a single solid equilibrium condition: as discussed below, more than one solid formation temperature can exist for mixtures. Finally, the software often requires excessive computation times and is not wellsuited to modern computers.

Table 1: Components available in KLSSP and the limits on mole fractions (in solvent or solute) and temperature that can be calculated for a given solute solid equilibrium within its model.

\begin{tabular}{c|cccc|ccc}
\multicolumn{3}{c}{} & \multicolumn{2}{c}{ Maximum } & & \multicolumn{2}{c}{ Maximum } \\
\cline { 1 - 3 } \cline { 5 - 7 } Component & $\mathrm{mol} \mathrm{mol}^{-1}$ & $\mathrm{~T} / \mathrm{K}$ & & & $\mathrm{mol} \mathrm{mol}^{-1}$ & $\mathrm{~T} / \mathrm{K}$ \\
Nitrogen & - & - & & n-Hexane & 0.2 & 150.0 \\
Methane & - & - & & n-Heptane & 0.05 & 166.0 \\
Ethane & - & - & & n-Octane & 0.05 & 190.7 \\
Propane & - & - & & n-Nonane & 0.1 & 190.7 \\
i-Butane & 0.4 & - & & n-Decane & 0.3 & 190.7 \\
n-Butane & 0.4 & - & & Benzene & 0.001 & 190.7 \\
i-Pentane & 0.3 & - & & Cyclohexane & 0.05 & - \\
n-Pentane & 0.3 & - & & Carbon Dioxide & 0.15 & 190.7
\end{tabular}

The KLSSP model was tuned to measured solid-liquid equilibrium (SLE) conditions for binary mixtures through the adjustment of four empirical parameters, two in a temperaturedependent solid phase fugacity function, and two in an activity coefficient function for the solute. This tuning ensured that the KLSSP model was essentially as accurate as the SLE data obtained for binary mixtures; however, such an empirical approach necessarily placed limits on the model's scope. Predictions of SLE in industrially important multi-component systems were made through the use of empirical mixing rules for each of the four parameters but the absence of a theoretical foundation significantly constrained the achievable accuracy.

In contrast, the semi-theoretical basis and broader generality of equations of state (EOS) make them, in principle, a better prospect for the basis of SLE models even if their domain is limited to the fluid phases. As shown below, the same EOS can be used to calculate both the 
solid phase fugacity (from the pure fluid model combined with some properties of the pure solid) and the partial fugacity coefficient of the solute in the liquid mixture. A dependence on pressure is implicitly included, mixing rules are available over the entire range of composition, and new compounds are readily incorporated into the model framework. Importantly, these EOS models are able to predict and distinguish between both SLE and solid-liquid-vapour equilibrium (SLVE) conditions for eutectic mixtures (i.e. where the solid phase is a pure substance).

Ideally, multi-parameter fundamental Helmholtz equations, such as the GERG-2008 or EOS-LNG models [24, 25] which aim to describe the available data for fluid phase properties within their experimental uncertainty could be used to predict solid-fluid equilibrium (SFE) in eutectic mixtures. However, the flexibility of these multi-parameter equations requires an extensive set of relevant and sufficiently accurate thermodynamic data be used in their development to avoid poor predictions beyond their range validity, as shown for example by Rowland et al. [26]. The simple functional form of cubic EOS and their ability to represent fluid phase equilibria in multi-component natural gas mixtures as accurately as the Helmholtz models make them a more robust choice currently for the basis of an improved SLE model to replace KLSSP. However, the cubic EOS implemented in most commercially available software packages used by industry are optimised, through the adjustment of the pure fluid and binary interaction parameters, to the description of vapour-liquid equilibrium data. Consequently, there is a large difference in the SFE predictions made with these default cubic EOS models and those made with KLSSP in regions of interest to the LNG industry.

Accordingly, we present here a new software tool, called ThermoFAST, which is based on a cubic EOS and has been designed to overcome the main limitations of KLSSP for the prediction of SFE in cryogenic natural gas processing. The optimized binary interaction parameters used within ThermoFAST were obtained by regression of the model to the SFE data available in the literature for relevant binary mixtures of light and heavy hydrocarbons or $\mathrm{CO}_{2}$. The resulting performance of ThermoFAST in the prediction of SFE conditions for binary and multi-component mixtures is better and more comprehensive than other available models; consequently, in 2018 the GPA Midstream Association endorsed ThermoFAST as the replacement of KLSSP for calculations of cryogenic solids formation in natural gas processing. Finally, after describing the model and demonstrating its performance relative to the available data, we use ThermoFAST to explore the nature of solid phase behavior in natural gas mixtures and predict unexpected transitions that, if verified experimentally, could 
potentially offer new methods of remediating solid formation events that occur in LNG production.

\section{Model Framework}

The majority of phase equilibria calculations carried out for natural gas process simulations are still based on a cubic EOS. A cubic EOS is recommended because it can be solved rapidly due to its fundamental simplicity and its accuracy for phase equilibria calculations in multi-component mixtures of hydrocarbons is generally as good as any other model. The Peng-Robinson (PR) EOS [29], which is the recommended cubic in ThermoFAST, has the following form for a pure fluid:

$$
\begin{gathered}
p=\frac{R T}{v-b}-\frac{a}{v(v+b)+b(v-b)} \\
a=0.45724 \frac{R^{2} T_{c}^{2}}{p_{c}} \alpha \\
\alpha=\left(1+\kappa\left(1-\sqrt{\frac{T}{T_{c}}}\right)\right)^{2} \\
\kappa=0.37464+1.54226 \omega-0.26992 \omega^{2} \\
b=0.07780 \frac{R T_{c}}{p_{c}}
\end{gathered}
$$

The PR EOS can be extended to the description of mixtures by using the following mixing rules for a set number of components, $N$, to generalise the $a$ and $b$ term in eq. (2.1) in terms of the component values, $a_{\mathrm{i}}$ and $b_{\mathrm{i}}$.

$$
\begin{gathered}
a=\sum_{i=1}^{N} \sum_{j=1}^{N} x_{i} x_{j}\left(1-k_{i j}\right) \sqrt{\left(a_{i}\right)\left(a_{j}\right)} \\
b=\sum_{i=1}^{n} x_{i} b_{i}
\end{gathered}
$$

Phase equilibrium calculations with an EOS are very sensitive to the values of the binary interaction parameter, $k_{i j}$, as presented in equation (2.6). As such the $k_{i j}$ is often treated as an adjustable parameter the value of which is derived empirically by forcing agreement between the measured VLE data and the corresponding EOS predictions. 
A key property in phase equilibrium calculations is the partial fugacity of a component which can be expressed via the following thermodynamic relationship,

$$
f_{i}=x_{i} P \exp \left(\int_{P_{0}}^{P}\left(\frac{\overline{v_{l}}}{R T}-\frac{1}{P}\right) d P\right)
$$

where the partial molar volume, $\bar{v}$ of component, $i$, can be calculated from the cubic EOS. Taking the reference pressure, $P_{0}$, to be zero, eq. (2.8) then becomes,

$$
\begin{aligned}
f_{i}=x_{i} P \exp \left[\frac{B_{i}}{B}(Z-1)-\ln (Z-B)\right. \\
\left.\quad+\frac{A}{2 \sqrt{2} B}\left[\frac{B_{i}}{B}-\frac{2}{a} \sum_{j=1}^{n} x_{j}\left(1-k_{i j}\right) \sqrt{a_{i} a_{j}}\right] \ln \left(\frac{Z+(\sqrt{2}+1) B}{Z-(\sqrt{2}-1) B}\right)\right]
\end{aligned}
$$

where,

$$
A=\frac{a P}{R^{2} T^{2}}, \quad B=\frac{b P}{R T}, \quad B_{i}=0.0788 \frac{P_{r, i}}{T_{r, i}}, \quad Z=\frac{P v}{R T}
$$

Central to the calculation of the conditions under which solids form in fluid mixtures is the solution of the phase equilibrium problem. The general solution to such problems where a variable number of phases coexist in a closed system at equilibrium, is determined by following three criteria which must be satisfied for each phase present (labelled by $k$ and $r$ ):

1. temperature in all phases is equal: $T_{r}=T_{k}$

2. pressure in all phases is equal: $P_{r}=P_{k}$

3. fugacity of each component in all phases is equal: $f_{i, r}=f_{i, k}$

where, $\quad k=1$ to $\pi(k \neq r)$,

$$
\begin{aligned}
& \pi=\text { number of phases, } \\
& i=1 \text { to } N \text {, and } \\
& N=\text { number of components. }
\end{aligned}
$$

For simple systems or calculations where the number and types of phases that are present at equilibrium are known or specified beforehand (e.g. vapour and liquid), conditions (1) to (3) can be solved rapidly without difficulty. On the other hand, if the number and types of phases present at equilibrium are not known prior to calculation, a fourth criterion - minimise the Gibbs Energy - can be introduced as was done by Gupta et al. (1991) [27] and Ballard et al. (2004) [28]. However, a disadvantage of this method is the requirement for good initial estimates of the unknowns (the amounts and compositions of each phase). 
Here a new method is described for solving the general temperature-pressure flash calculation, which does not require such stringent initial estimates of every phase's properties. The method, which is implemented within the ThermoFAST software package, utilises a Newton-Raphson based approach similar to those developed by Gupta et al. (1991) [27] and Ballard et al. (2004) [28]; however it requires only initial estimates of how the components partition across any vapour and liquid phases present, which can be done with well-established correlations. This also facilitates the addition of new components into such calculations without the need to develop new correlations for estimating how they partition across all phases that might be present.

\subsection{Calculating Component Fugacities in Fluid and Solid Phases}

For a flash calculation at a specified temperature and pressure, criteria (1) and (2) are automatically satisfied and therefore the task becomes to find the solution to the fugacity criterion. To determine the solubility of a component in a fluid mixture, the partial fugacity of that component calculated using eq (2.9) must be compared with the fugacity of that component in the solid phase. The SFE model within ThermoFAST is based on the assumption that any solid phase present consists of a pure substance although multiple (pure) solid phases can be present simultaneously. This assumption is reasonable for hydrocarbons or solid $\mathrm{CO}_{2}$ although it does not apply to gas hydrates, which are currently excluded from consideration. The fugacity of pure component $i$ in the solid phase, $f_{i}^{s}$, is calculated using an equation first derived by Hildebrand and Scott [30] and endorsed by Prausnitz [31],

$$
\begin{aligned}
\ln \left(f_{i}^{S}\right) & =\ln \left(\varphi_{\text {pure }, i}^{L} P\right)-\frac{\Delta H_{f, i}}{R T_{m, i}}\left[\frac{T_{m, i}}{T}-1\right]+\frac{\Delta c_{p, i}^{L \rightarrow S}}{R}\left[\frac{T_{m, i}}{T}-1+\ln \left(\frac{T}{T_{m, i}}\right)\right] \\
& -\frac{\Delta v_{i}^{L \rightarrow S}\left(P-P_{m}\right)}{R T}
\end{aligned}
$$

Here, $\varphi_{\text {pure }, i}^{L}$ is the fugacity coefficient of pure component $i$ in the liquid phase, (also calculated using the PR EOS); $T_{m, i}$ is the melting temperature of pure component $i ; \Delta H_{f, i}$ is the corresponding molar heat of fusion at $T_{m, i} ; \Delta c_{p, i}^{L \rightarrow S}$ is the molar specific heat of the component as a pure liquid minus that of the pure solid $i$, which is approximated as being independent of temperature; $\Delta v_{i}^{L \rightarrow S}$ is the change in volume between the liquid and solid phase of component $i$; and $P_{m}$ is a reference pressure for the given melting temperature, 
which in this case is atmospheric pressure. Each term on the right of eq (2.14) is calculable from knowledge of the pure component properties, which were taken from various sources including the DIPPR ${ }^{\circledR}$ database [32-35]. A slight modification to equation (2.14) is needed if the solid undergoes a crystalline phase transition at a temperature below $T_{\mathrm{m}}$, as is the case for cyclohexane. This modification is discussed further in Section 3.1.

\subsection{System of Equations for Flash Calculations}

The following set of equations describes the material balance for a system of $\mathrm{N}$ components distributed across $\pi$ possible phases,

$$
\sum_{k=1}^{\pi} \alpha_{k} x_{i k}=z_{i}, \quad i=1, \ldots, N
$$

where, $\alpha_{k}$ is the normalised phase fraction of phase $k$ and $x_{i k}$ is the mole fraction of component $i$ in phase $k$. Particularly when more than three phases are present, it is efficient to specify a reference phase, which must contain all of the components (even in trace concentrations) at equilibrium: accordingly the reference phase should be a fluid, a liquid phase is selected by default unless the results of an initial VLE(-only) flash indicate that only a vapour phase is present. By considering the reference phase, $r$, eq. (2.15) can be re-written as

$$
\alpha_{r} x_{i r}+\sum_{\substack{k=1 \\ k \neq r}}^{\pi} \alpha_{k} x_{i k}=z_{i}, \quad i=1, \ldots, N
$$

For a closed system, material balance imposes two further constraints on the phase fractions and component mole fractions, respectively,

$$
\begin{gathered}
\alpha_{r}=1-\sum_{\substack{k=1 \\
k \neq r}}^{\pi} \alpha_{k}, \quad k=1, \ldots, \pi, \\
\sum_{\substack{k=1 \\
k \neq r}}^{\pi} x_{i k}=1, \quad k=1, \ldots, \pi .
\end{gathered}
$$

The requirement that each component's partial fugacity in each phase be equal at equilibrium means that, 


$$
\frac{f_{i r}}{f_{i k}}=\frac{x_{i r} \phi_{i r} P}{x_{i k} \phi_{i k} P}=1, \quad i=1, \ldots, N, \quad k=1, \ldots, \pi
$$

where $f_{i k}$ and $\phi_{i k}$ are the partial fugacity and partial fugacity coefficient of component, $i$, in phase, $k$, respectively. This representation of the third criterion, eq. (2.13), allows the problem to be conveniently recast in terms of calculating the equilibrium (or partition) coefficients, $K_{i k} \equiv x_{i k} / x_{i r}$ for component $i$ across phases $k$ and $r$. With initial estimates of $x_{i r}$ and $K_{i k}$, the compositions of other phases present can be estimated by solving the material balance equations (2.16) to (2.18). New estimates of the equilibrium coefficients can then be generated using equations (2.9) and (2.14) for any fluid and solid phases present, respectively, via:

$$
K_{i k}=\frac{\phi_{i r}}{\phi_{i k}}=\frac{x_{i k}}{x_{i r}} \frac{f_{i r}}{f_{i k}}, \quad i=1, \ldots, N, \quad k=1, \ldots, \pi
$$

An iterative procedure is then followed until the calculated phase compositions and fractions converge within a specified tolerance. The remaining key steps, then, are achieving sufficiently good initial estimates of $K_{i k}$ and establishing which phases are stable and hence present at equilibrium.

\subsection{Solving the Flash Calculation via Successive Substitution}

Figure 1 shows the logical flow of the primary algorithm used by ThermoFAST to solve the majority or all of the flash calculations. The algorithm works by performing an initial VLE-only flash, using well-established correlations [36] to provide initial estimates for the $K_{i k}$ of the fluid phases. Since all solid phases are pure compounds and the composition of the reference phase has been estimated using the initial VLE-only flash, calculation of the solid phase fugacity using eq (2.14) for each component at the specified temperature and pressure provides an initial estimate of the $K_{i k}$ for the solid phase(s). These then allow a solid-liquid equilibrium flash calculation to provide an estimate of the phase fractions $\alpha_{k}$ : stable phases have $\alpha_{k}>0$.

This procedure is iterated until either convergence is achieved in the $x_{i k}$ and $\alpha_{k}$, or the specified maximum number of iterations is reached. Often the tolerance criterion is achieved within three iterations and the maximum number of iterations is set to five. If this condition is reached then the outputs of the (unconverged) calculation are used as improved initial estimates within a more complicated numerical algorithm based on the work of Gupta et al. (1991) [27] and Ballard et al. (2004) [28], which incorporates a Newton-Raphson algorithm 
to achieve a rapid convergence, rather than the slower (but less sensitive) successive substitution method used for the algorithm shown in Figure 1. Further detail about this algorithm and its implementation in ThermoFAST is given in Baker (2018) [37].

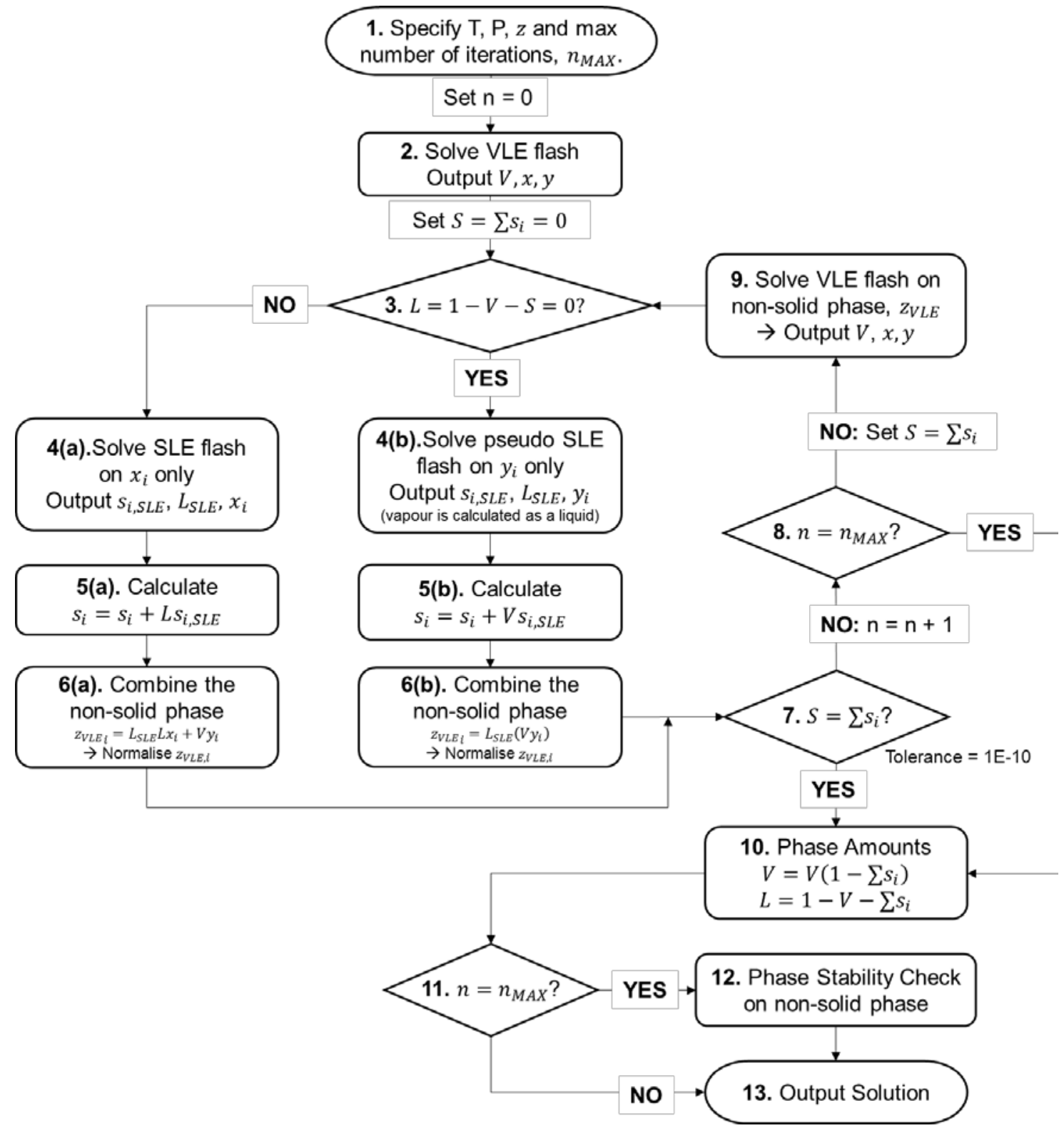

Figure 1: ThermoFAST's isothermal algorithm for solving solid-liquid-vapour equilibrium using a successive substitution method. The VLE and SLE flash algorithms are given in Baker (2018) [37]. Here, $T$ is temperature, $P$ is pressure, $z$ is overall composition, $i$ represents a particular single component, $f$ is fugacity, $s$ is the solid composition, $x$ is liquid composition, $y$ is vapour composition, $\varphi^{L}$ is the partial fugacity in the liquid phase, $V$ is the overall vapour mole fraction, $L$ is the overall liquid mole fraction, $\mathrm{n}$ is the number of iterations, $n_{\max }$ is the maximum number of iterations, and the subscript SLE refers to any variable determined from the SLE Flash algorithm within the multiphase equilibrium calculation as described in Baker et al. (2018) [38]. The phase stability check is performed by calculating the bubble point, $T_{b u b}^{V L E}$, on the non-solid phase and if $T_{b u b}^{V L E}<T$ then $V=0$ and $L=L+$ $V$, otherwise $V>0$ (and if vapour did not exist then $V=L$ and $L=0$ ). $T_{b u b}^{V L E}$ is calculated from the algorithm provided by Baker (2018) [37]. 


\section{Comparison with Experimental Data}

The PR EOS implemented in ThermoFAST has been tuned to solid-fluid equilibrium data measured for 58 binary systems relevant to LNG and natural gas production. The binary interaction parameters $k_{\mathrm{ij}}$ in eq. (3.4) were adjusted using the Levenberg-Marquardt nonlinear least squares regression method to minimise the following objective function,

$$
\text { ObjFunc }=\sum_{i=1}^{N}\left(T_{i, \text { meas }}-T_{i, \text { calc }}\right)^{2}
$$

where, $i$ is an index over the $N$ data points available for a given binary mixture, $T_{i, \text { meas }}$ is the measured solid-fluid equilibrium temperature, and $T_{i, c a l c}$ is the solid-fluid equilibrium temperature calculated using ThermoFAST. The binary systems considered consisted of pairs of the following compounds: alkanes from methane to n-decane (including isobutane, isopentane, and neo-pentane), $\mathrm{CO}_{2}$, cyclopentane, cyclohexane, methyl-cyclohexane, and the aromatics benzene, toluene, ethylbenzene, m-xylene, o-xylene, and p-xylene. Of the possible binary pairs from this list of compounds, only 58 systems had sufficient quality data to reliably tune a $k_{\mathrm{ij}}$ optimised for solid-fluid equilibrium predictions. For solid-fluid equilibrium calculations involving any of the remaining pairs, the EOS in ThermoFAST uses a single binary interaction parameter optimised for VLE predictions [37].

Table 2 shows a summary of the performance of the tuned ThermoFAST and KLSSP models in representing the available experimental data. Three different scenarios were used to quantify the performance of each model in predicting measured melting temperature: (1) all 58 binary mixtures over the entire experimental range, (2) the binary mixtures that contain components available within KLSSP and (3) binaries that are available and within KLSSP's range of validity as per Table 1 . The standard user interface available for KLSSP does not allow calculations outside its range of validity; however the Visual Basic interface to KLSSP implemented within Microsoft Excel allows these limits to be bypassed. 
Table 2: Summary of ThermoFAST and KLSSP predictions for the available literature data for solid equilibria in binary mixtures according to three criteria: (A) all 58 binary systems, (B) 36 binary systems that can be calculated using KLSSP and (C) 17 binary systems that are available in KLSSP and are within KLSSP's recommended range of validity as per Table 1. Shown is the average, minimum and maximum of the root-mean-squared temperature deviations (rmsd) between the model and literature data, as well as the standard deviation, $\sigma_{x}$, and the median. For case (A) the performance of an un-tuned ThermoFAST model with $k_{i j}=0$ is shown.

\begin{tabular}{|c|c|c|c|c|c|c|}
\hline \multirow{2}{*}{ CRITERIA } & \multirow{2}{*}{ MODELS } & \multicolumn{3}{|c|}{ rmsd / K } & \multirow{2}{*}{$\begin{array}{l}\sigma_{\mathrm{x}} \\
/ \mathrm{K}\end{array}$} & \multirow{2}{*}{$\begin{array}{c}\text { Median } \\
\text { / K }\end{array}$} \\
\hline & & Avg. & Min. & Max. & & \\
\hline \multirow{2}{*}{$\begin{array}{c}\text { (A) } \\
\text { all binary mixtures }\end{array}$} & ThermoFAST & 1.5 & 0.1 & 5.5 & 1.3 & 1.1 \\
\hline & ThermoFAST (set $\left.k_{i j}=0\right)$ & 11.1 & 0.2 & 60.6 & 13.6 & 4.7 \\
\hline \multirow{2}{*}{$\begin{array}{l}\text { (B) } \\
\text { binaries limited to } \\
\text { components in } \\
\text { Table } 1\end{array}$} & ThermoFAST & 1.4 & 0.1 & 4.3 & 1.2 & 1.0 \\
\hline & KLSSP & 19.1 & 0.5 & 64.0 & 18.9 & 9.0 \\
\hline \multirow{2}{*}{$\begin{array}{c}\mathbf{( C )} \\
\text { binaries and } \\
\text { compositions } \\
\text { limited to Table } 1\end{array}$} & ThermoFAST & 1.2 & 0.2 & 5.2 & 1.2 & 0.8 \\
\hline & KLSSP & 5.6 & 0.5 & 16.2 & 5.1 & 3.6 \\
\hline
\end{tabular}

Table 2 shows that by tuning the $k_{\mathrm{ij}}$ the models implemented in ThermoFAST can represent all of the data for all binaries with an average rmsd of $1.5 \mathrm{~K}$. Using KLSSP outside its stated range of validity produces an average rmsd almost twice as large as that achieved with the un-tuned PR EOS model (i.e. set $k_{\mathrm{ij}}=0$ ), which reflects the general advantage of using an equation of state over an activity coefficient model. Within its range of validity, the average rmsd of the KLSSP model is $4.4 \mathrm{~K}$ larger than that of the tuned ThermoFAST model. Thus not only does the ThermoFAST model improve upon KLSSP's range of validity in terms of conditions and compounds considered, it also describes more accurately those data that are available but outside the range of validity. In the following two subsections graphical comparisons are presented for a selection of binary and multi-component mixtures where data relevant to gas processing are available. Further graphical comparisons to experimental data for mixtures not included here are presented by Baker (2018) [37]. 


\subsection{Exemplar Binary Mixtures}

Figure 2 to Figure 7 show the solid-fluid equilibrium predictions of ThermoFAST and KLSSP together with available experimental data for the following binary mixtures containing methane or ethane with benzene, cyclohexane, toluene, neopentane and $\mathrm{CO}_{2}$, respectively. ThermoFAST predictions are shown as blue circles, data sets are presented by dark grey symbols and KLSSP predictions are represented as a continuous line as it has no pressure dependence for binary systems.

In the methane + benzene system shown by Figure 2, KLSSP clearly represents the available data well at the low concentrations of benzene that are of relevance to LNG production. It appears to have been tuned to slightly under-predict the equilibrium temperature probably to ensure any error produced was of a conservative nature. ThermoFAST is also able to represent the data well but over the entire range of compositions for which measurements exist. Within its recommended limits, KLSSP represents the data with an rmsd of $8.4 \mathrm{~K}$ while ThermoFAST has an rmsd of only $1.5 \mathrm{~K}$. 

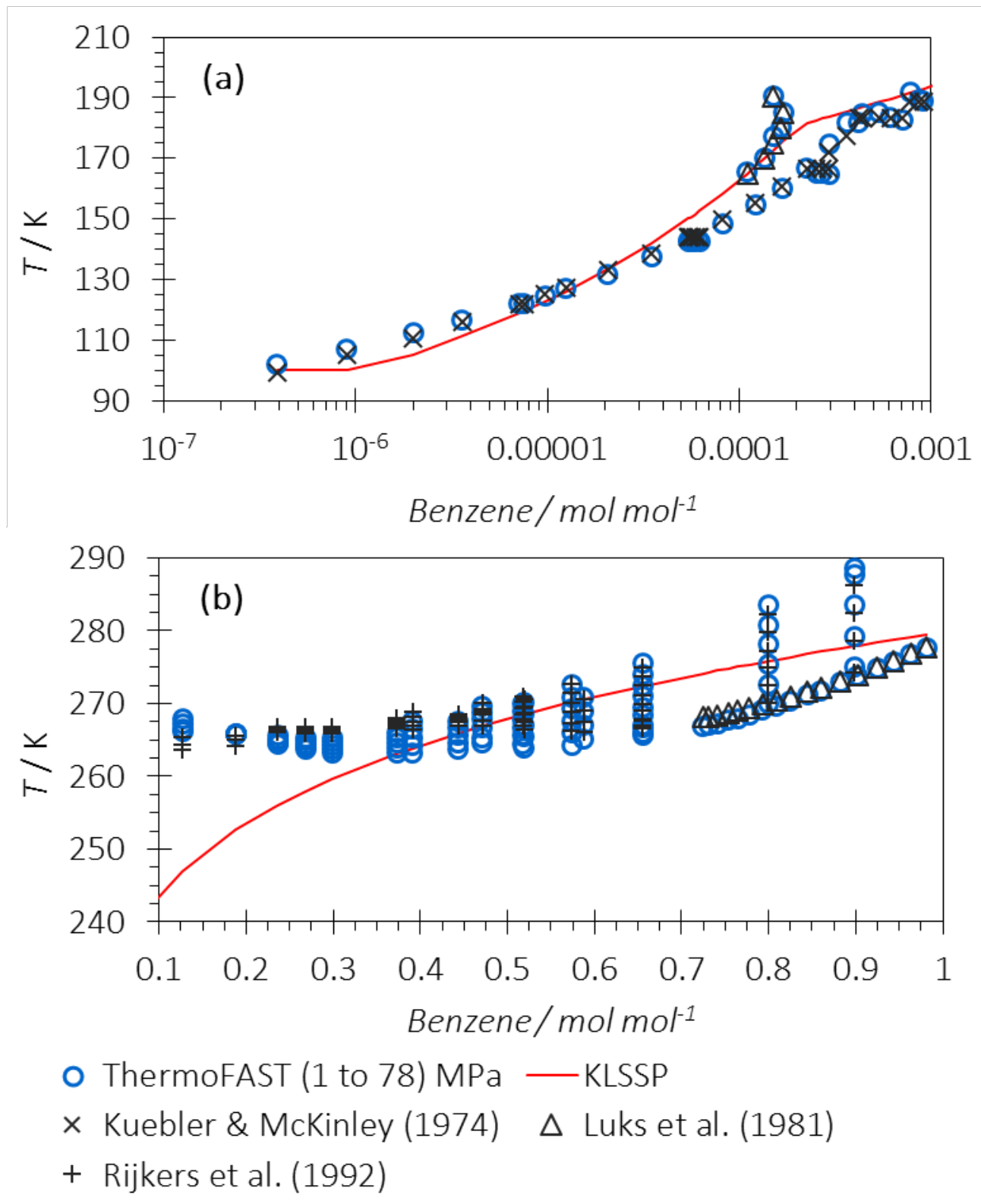

Figure 2: Solid-fluid equilibrium diagram for methane + benzene comparing ThermoFAST and KLSSP predictions against experimental data $[19,39,40]$ at (a) low and (b) high concentrations of benzene. ThermoFAST uses the experimental pressure as an input together with the composition.

For systems containing cyclohexane, it is necessary to consider the solid-solid crystalline transition described by Parks et al. [41] at $185.9 \mathrm{~K}$ and atmospheric pressure. For any mixture containing cyclohexane that is at a temperature below the crystalline phase transition of pure cyclohexane $\left(T_{S 1-S 2}=185.9 \mathrm{~K}\right)$ it is necessary to use a modified version of equation (2.14),

$$
\begin{aligned}
\ln \left(f_{i}^{S}\right) & =\ln \left(\varphi_{\text {pure, }}^{L} P\right)-\frac{\Delta H_{f, i}}{R T_{m, i}}\left[\frac{T_{m, i}}{T}-1\right]-\frac{\Delta H_{(S 1-S 2), i}}{R T_{(S 1-S 2), i}}\left[\frac{T_{(S 1-S 2), i}}{T}-1\right] \\
& +\frac{\Delta c_{p, i}^{L \rightarrow S}}{R}\left[\frac{T_{m, i}}{T}-1+\ln \left(\frac{T}{T_{m, i}}\right)\right]-\frac{\Delta v_{i}^{L \rightarrow S}\left(P-P_{m}\right)}{R T}
\end{aligned}
$$


This equation assumes (1) the heat capacity change between the different crystalline structures is negligible, and (2) the pressure effect on the crystalline transition temperature is negligible. For cyclohexane, the enthalpy of the crystalline phase transition, $\Delta \boldsymbol{H}_{(\boldsymbol{S 1} \mathbf{S} \mathbf{S}), \boldsymbol{i}}=$ $6.715 \mathrm{~kJ} \cdot \mathrm{mol}^{-1}$. Figure 3 shows solid-fluid equilibrium data for $\mathrm{n}$-hexane + cyclohexane measured at atmospheric pressure [42]. Cyclohexane's solid-solid transition causes the temperature-solubility curve to have a slope discontinuity at $185.9 \mathrm{~K}$ and a cyclohexane mole fraction of 0.6. The use of eq (3.2) for mixtures containing cyclohexane allows ThermoFAST to represent the experimental data with an rmsd of only $0.18 \mathrm{~K}$.

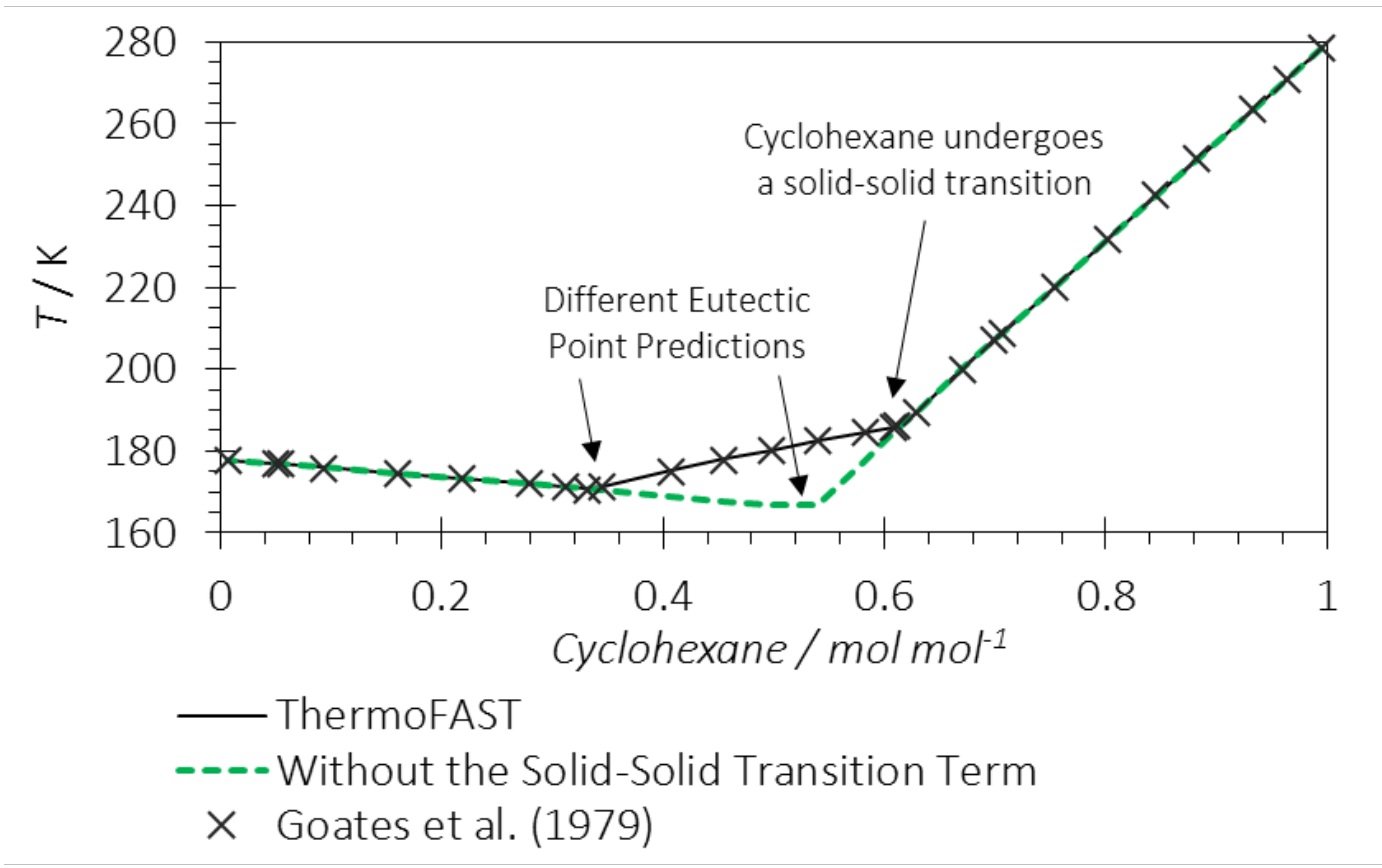

Figure 3: Solid-fluid equilibrium diagram for $n$-hexane + cyclohexane comparing the predictions of the models in ThermoFAST with (eq (3.2)) and without eq (2.14) the solid-solid transition term, against experimental data [42] measured at atmospheric pressure.

Figure 4 shows solid-fluid equilibrium data for the system methane + cyclohexane. The KLSSP predictions are generally reasonable although it is unable to represent the pressure dependence of certain data even within its range of validity as shown in Figure 4 (a). In contrast ThermoFAST is able to capture the large changes in the solid-fluid equilibrium temperature that occur with pressure and cyclohexane concentration, with an rmsd of only $2.0 \mathrm{~K}$. 

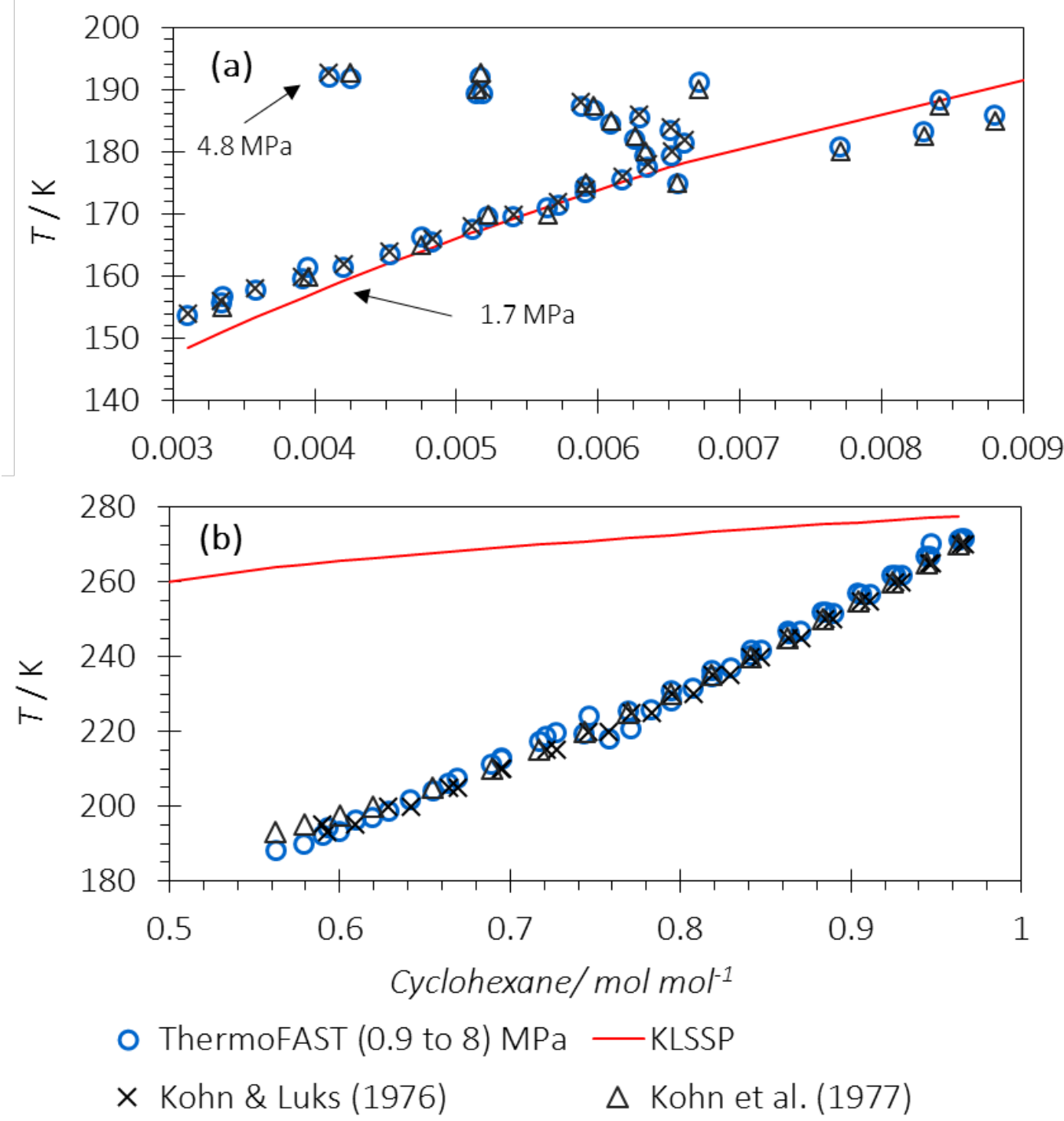

Figure 4: Solid-fluid equilibrium diagram for methane + cyclohexane comparing ThermoFAST and KLSSP predictions against experimental data $[10,22]$ at (a) low and (b) high concentrations of cyclohexane. ThermoFAST uses the experimental pressure as an input together with the composition.

Two binary systems not described by KLSSP because they contain toluene and neopentane, respectively, are shown in Figure 5 and Figure 6. ThermoFAST represents the experimental data for the system methane + toluene (Figure 5) with an rmsd of only $1.4 \mathrm{~K}$. Figure 6 shows the results for the methane + neopentane system for which only three data points are available. These solid-fluid equilibrium data were measured along the system's bubble-point curve; the ThermoFAST user interface allows the user to specify solid-fluid equilibrium temperatures should be calculated according to this constraint. The dependence of melting temperature with neopentane concentration is not represented as well as for most binary systems. This result provides further motivation to acquire additional solid-fluid equilibrium data for methane + neopentane binary. 


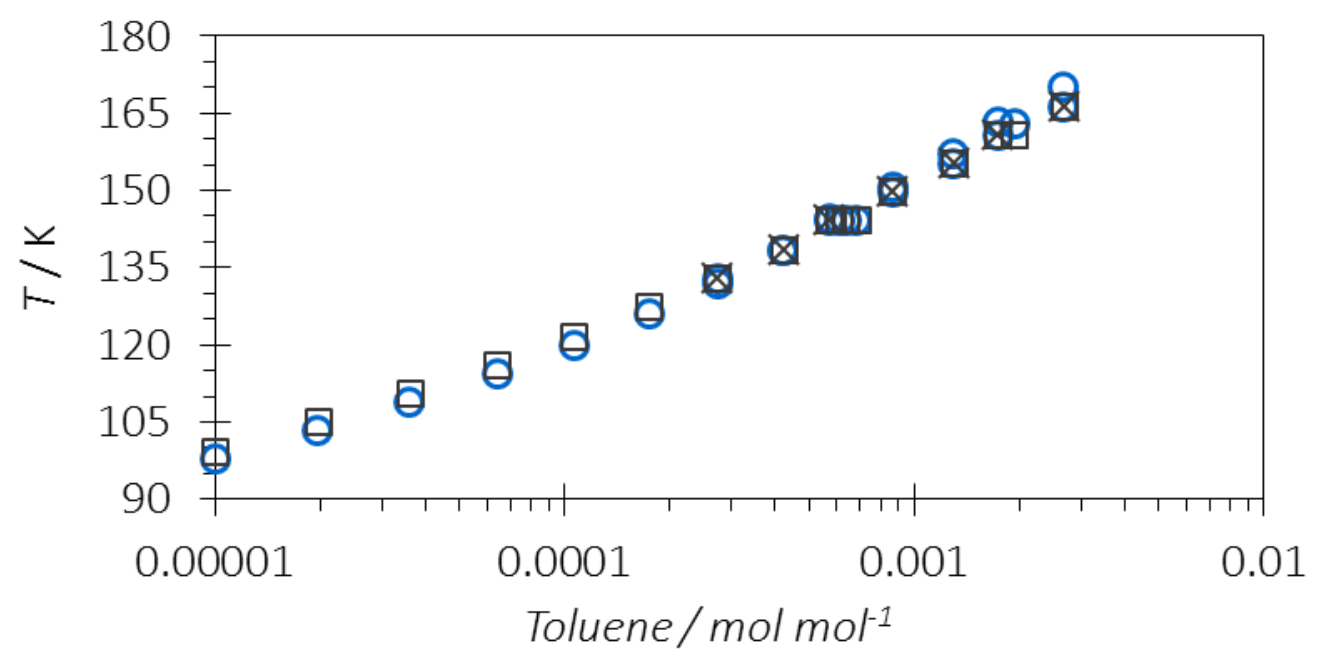

OThermoFAST (0.4 to 13) MPa $\quad \square$ Kuebler \& McKinley (1974)

$\times$ Luks et al. (1975)

Figure 5: Solid-fluid equilibrium diagram for methane + toluene comparing ThermoFAST predictions against experimental data [21,39] with respect to the absolute temperature. ThermoFAST uses the experimental pressure as an input together with the composition.

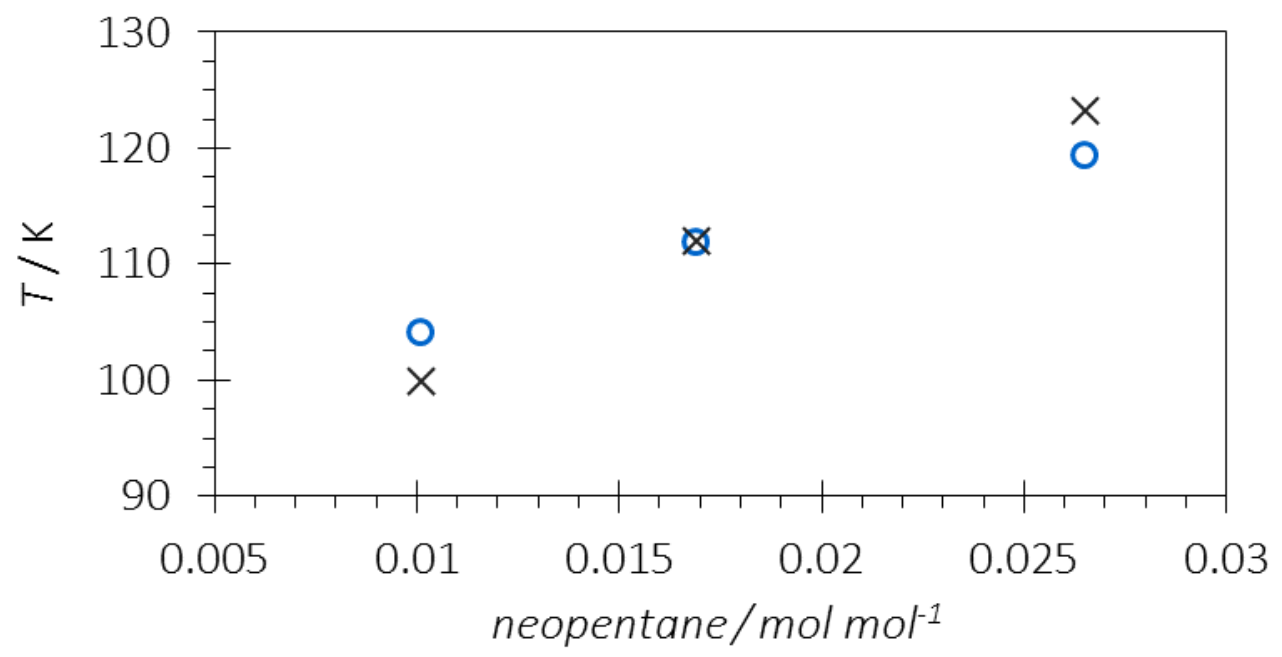

o ThermoFAST (1.7 to 1.8) MPa $\quad \times$ Preston et al. (1971)

Figure 6: Solid-fluid equilibrium diagram for methane + neopentane comparing ThermoFAST predictions against the limited available data [43], which were measured along the bubble point curve.

Figure 7 shows ThermoFAST is able to represent the experimental data available for the methane $+\mathrm{CO}_{2}$ binary system very well across the complete range of $\mathrm{CO}_{2}$ fraction. The largest deviations to the experimental data for both ThermoFAST and KLSSP occur for the data of Pikaar [44], where deviations of up to $14 \mathrm{~K}$ and $23 \mathrm{~K}$ occur for ThermoFAST and KLSSP, respectively. Although shown in Figure 7, this data set has been excluded from tuning as the internal consistency and repeatability are poor. At the low $\mathrm{CO}_{2}$ concentrations 
relevant to LNG production and within KLSSP's range of validity, the tuned ThermoFAST model has an rmsd of only $2.2 \mathrm{~K}$, compared with $16.4 \mathrm{~K}$ for the KLSSP model. Including the 41 additional conditions measured by Pikaar [44] at low $\mathrm{CO}_{2}$ concentrations, the overall rmsd of the models over this range increases to $3.0 \mathrm{~K}$ and $16.2 \mathrm{~K}$ for ThermoFAST and KLSSP, respectively.

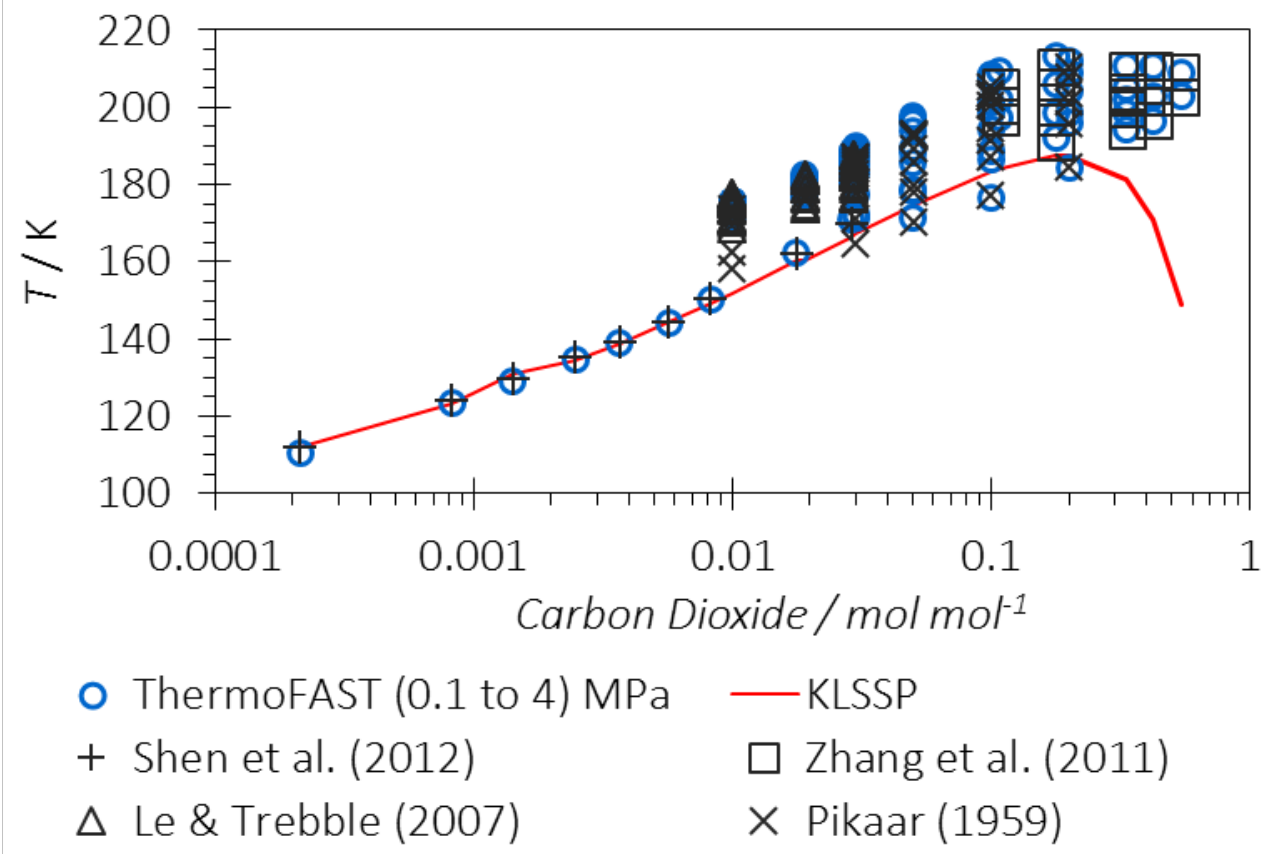

Figure 7: Solid-fluid equilibrium diagram for methane + carbon dioxide comparing ThermoFAST and KLSSP predictions against experimental data [44-47]. ThermoFAST uses the experimental pressure as an input together with the composition.

\subsection{Exemplar Multicomponent Mixtures}

Table 3 summarises the comparisons of ThermoFAST and KLSSP predictions with data available for five multicomponent (MC) mixtures containing hexane, heptane, octane, benzene and cyclohexane. The ThermoFAST predictions have an rmsd of less than $3 \mathrm{~K}$. Figure 8 to Figure 10 show comparisons for some of the MC mixtures containing octane, benzene and cyclohexane solutes, respectively. Overall, ThermoFAST improved solid-fluid equilibrium predictions over those of KLSSP by a factor of 6.8 for multicomponent mixtures. The ThermoFAST predictions are shown as blue circles, data sets are presented by dark grey symbols and the KLSSP predictions are represented as red squares. 
Table 3: Summary of ThermoFAST and KLSSP predictions for available multicomponent solid-fluid equilibrium data. Shown is the average of the root-mean-squared temperature deviations (rmsd) between the model and literature data, as well as the ranges of experimental pressure and methane mole fraction.

\begin{tabular}{|c|c|c|c|c|c|c|}
\hline & \multicolumn{2}{|c|}{$\mathrm{rmsd} / \mathrm{K}$} & \multicolumn{2}{|c|}{$\mathrm{P} / \mathrm{MPa}$} & \multicolumn{2}{|c|}{$\mathrm{CH}_{4} / \mathrm{mol} \mathrm{mol}^{-1}$} \\
\hline & 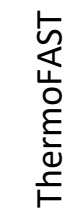 & $\begin{array}{l}\hat{\tilde{n}} \\
\underline{\tilde{y}}\end{array}$ & $\stackrel{x}{a}$ & $\risingdotseq$ & $\stackrel{x}{a}$ & $\stackrel{\S}{\Sigma}$ \\
\hline $\begin{array}{l}\mathrm{C}_{1}+\mathrm{C}_{2}+\mathrm{C}_{3} \\
\text { Solid: } \mathrm{nC}_{6} \mathrm{H}_{14} \\
\end{array}$ & 2.9 & 8.0 & 0.9 & 0.1 & 0.846 & 0.346 \\
\hline $\begin{array}{l}\mathrm{N}_{2}+\mathrm{C}_{1}+\mathrm{C}_{2}+\mathrm{C}_{3}+\mathrm{nC}_{4} \\
\text { Solid: } \mathrm{nC}_{7} \mathrm{H}_{16}\end{array}$ & 1.0 & 20.7 & 2.7 & 0.8 & 0.949 & 0.329 \\
\hline $\begin{array}{l}\mathrm{N}_{2}+\mathrm{C}_{1}+\mathrm{C}_{2}+\mathrm{C}_{3}+\mathrm{iC} 4+\mathrm{nC}_{4}+\mathrm{nC}_{6}+\mathrm{nC}_{7} \\
\text { Solid: } \mathrm{nC}_{8} \mathrm{H}_{18}\end{array}$ & 1.6 & 10.0 & 5.1 & 0.1 & 0.966 & 0.036 \\
\hline $\begin{array}{l}\mathrm{C}_{1}+\mathrm{C}_{2}+\mathrm{C}_{3}+\mathrm{iC} 4+\mathrm{nC}_{4} \\
\text { Solid: } \mathrm{C}_{6} \mathrm{H}_{6} \text { (benzene) }\end{array}$ & 2.1 & 9.9 & 16.0 & $<0.1$ & 0.891 & 0.036 \\
\hline $\begin{array}{l}\mathrm{C}_{1}+\mathrm{C}_{2}+\mathrm{C}_{3}+\mathrm{iC} 4+\mathrm{nC}_{4} \\
\text { Solid: } \mathrm{C}_{6} \mathrm{H}_{12} \text { (cyclohexane) }\end{array}$ & 2.5 & 20.4 & 3.8 & 0.1 & 0.888 & 0.040 \\
\hline
\end{tabular}

Figure 8 shows comparisons of KLSSP and ThermoFAST predictions to experimental data for a MC mixture with octane as the solute in solvents containing varying concentrations of $\mathrm{N}_{2}, \mathrm{CH}_{4}, \mathrm{C}_{2} \mathrm{H}_{6}, \mathrm{C}_{3} \mathrm{H}_{8}, i \mathrm{C}_{4} \mathrm{H}_{10}, n \mathrm{C}_{4} \mathrm{H}_{10}, n \mathrm{C}_{6} \mathrm{H}_{14}$ and $n \mathrm{C}_{7} \mathrm{H}_{16}$ at pressures between $(0.1$ and 5.1) MPa. ThermoFAST agrees well with the experimental data across the entire composition and pressure range with an overall rmsd of only $1.6 \mathrm{~K}$. For the data sets measured through the GPA research program, KLSSP does a good job at representing solubilities at low to moderate concentrations. However, KLSSP deviates by up to $30 \mathrm{~K}$ from the low concentration data of Chen et al. [17], with an overall rmsd of $10.0 \mathrm{~K}$ for all data. 


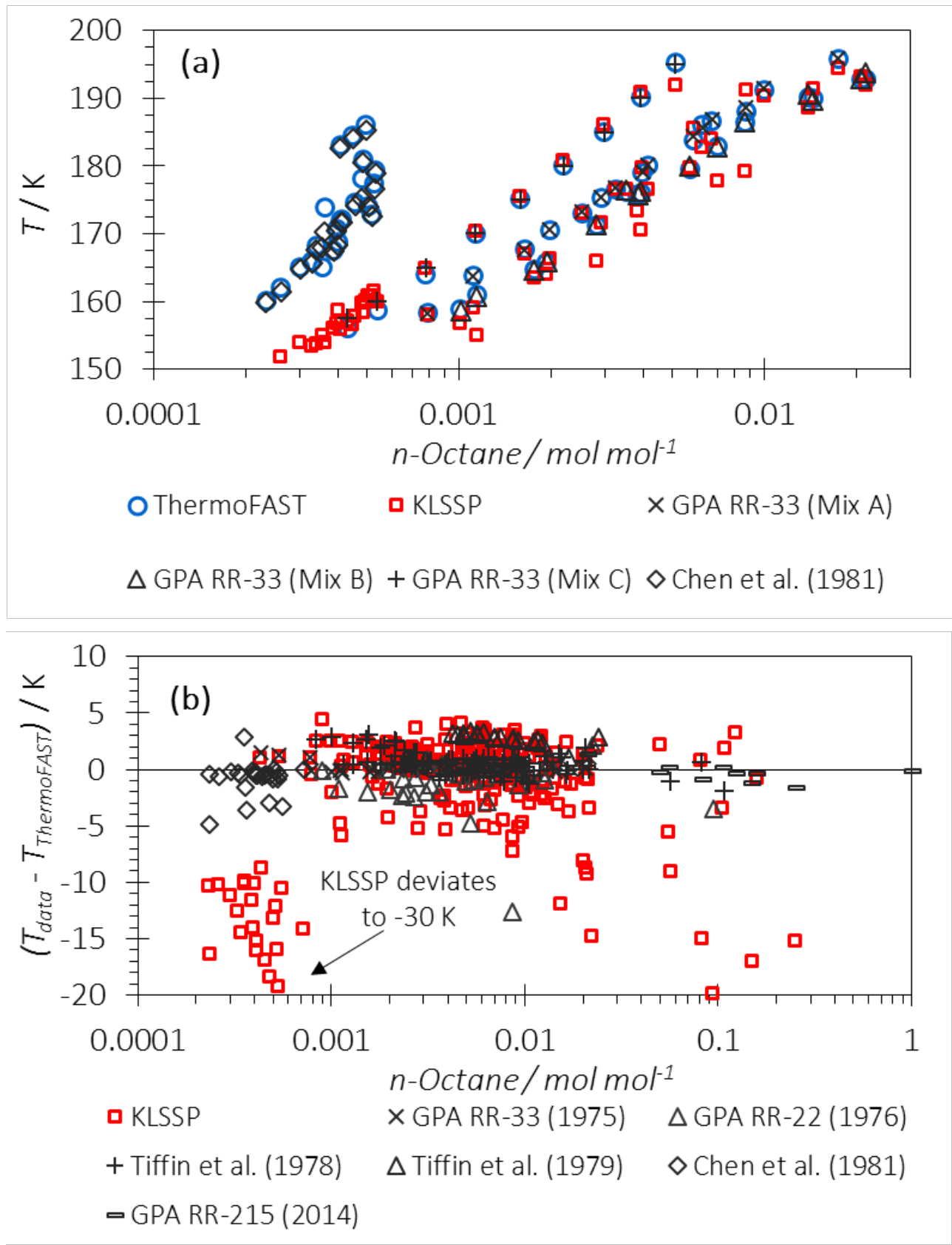

Figure 8: (a) Solid-fluid equilibrium data for a range of multicomponent systems containing octane as the solute and the corresponding predictions of ThermoFAST and KLSSP. Mix A is a $\mathrm{N}_{2}, 0.89 \mathrm{CH}_{4}$ to $0.02 \mathrm{nC}_{4} \mathrm{H}_{10}$; Mix B is a $0.87 \mathrm{CH}_{4}$ to $0.02 \mathrm{nC}_{6} \mathrm{H}_{14}$; Mix C is a $0.94 \mathrm{CH}_{4}$ to $0.02 \mathrm{C}_{3} \mathrm{H}_{8}$. (b) Deviations of melting temperatures predicted by ThermoFAST and KLSSP from all the experimental data $[12,15,17$, 21-23]. For clarity, only some of the data points represented in (b) are shown in (a).

Figure 9 shows comparisons of KLSSP and ThermoFAST predictions to experimental data for benzene in solvents containing varying concentrations of $\mathrm{CH}_{4}, \mathrm{C}_{2} \mathrm{H}_{6}, \mathrm{C}_{3} \mathrm{H}_{8}, i \mathrm{C}_{4} \mathrm{H}_{10}$ and $n \mathrm{C}_{4} \mathrm{H}_{10}$ at pressures varying between (0.0 and 16.0) MPa. ThermoFAST has a good agreement across the entire data range with an rmsd of only $2.1 \mathrm{~K}$. In contrast the KLSSP predictions of melting temperature are systematically low across the entire benzene concentration range with an rmsd of $9.9 \mathrm{~K}$. At certain low benzene concentrations, the 
KLSSP predictions are in good agreement with the data; however deviations as large as $24 \mathrm{~K}$ also occur in this region.

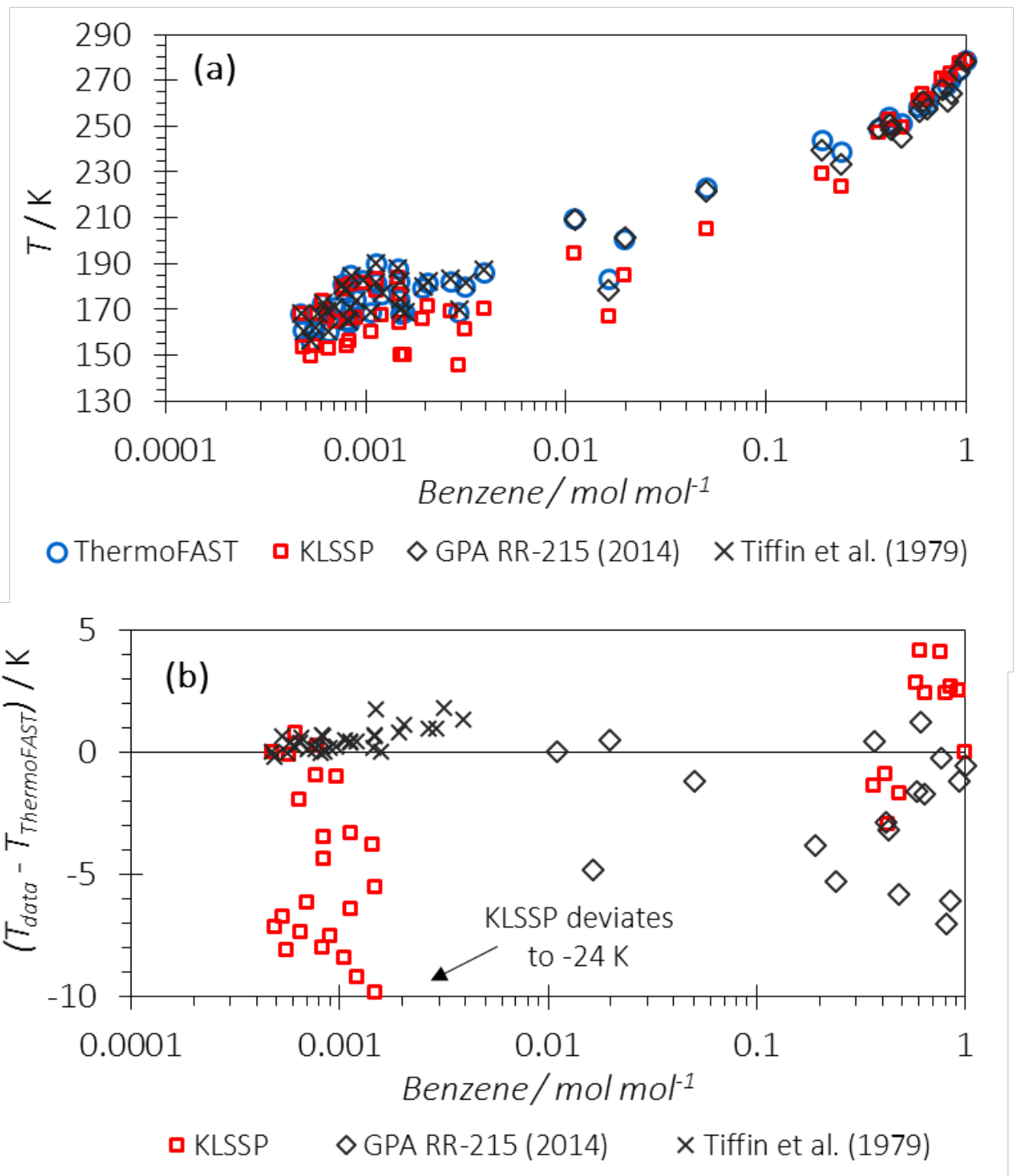

Figure 9: (a) Solid-fluid equilibrium data for a range of multicomponent systems containing benzene as the solute and the corresponding predictions of ThermoFAST and KLSSP. (b) Deviations of melting temperatures predicted by ThermoFAST and KLSSP from all the experimental data [14, 23]. For clarity, only some of the data points represented in (b) are shown in (a).

Figure 10 shows comparisons of KLSSP and ThermoFAST predictions to experimental data for cyclohexane in solvents containing varying concentrations of $\mathrm{CH}_{4}, \mathrm{C}_{2} \mathrm{H}_{6}, \mathrm{C}_{3} \mathrm{H}_{8}$, $i \mathrm{C}_{4} \mathrm{H}_{10}$ and $n \mathrm{C}_{4} \mathrm{H}_{10}$ at pressures varying between (0.1 and 3.8) MPa. The ThermoFAST predictions are in excellent agreement with the experimental data, with an rmsd of $2.5 \mathrm{~K}$. At temperatures near pure cyclohexane's crystalline phase transition (185.9 K at atmospheric pressure), the deviations from the experimental data increase to $12 \mathrm{~K}$. This might be an 
indication that the crystalline phase transition temperature has a strong dependence on pressure; however it could also reflect an increased uncertainty in the measurements made around these conditions. Unfortunately no other data exist to confirm either of these possibilities. In contrast, KLSSP provides a poor representation of the datasets with an rmsd of $16.8 \mathrm{~K}$ over the entire range of cyclohexane concentration. Even at low concentrations of cyclohexane it deviates by as much as $7 \mathrm{~K}$; this increases to $60 \mathrm{~K}$ at temperatures close to cyclohexane’s crystalline phase transition. 

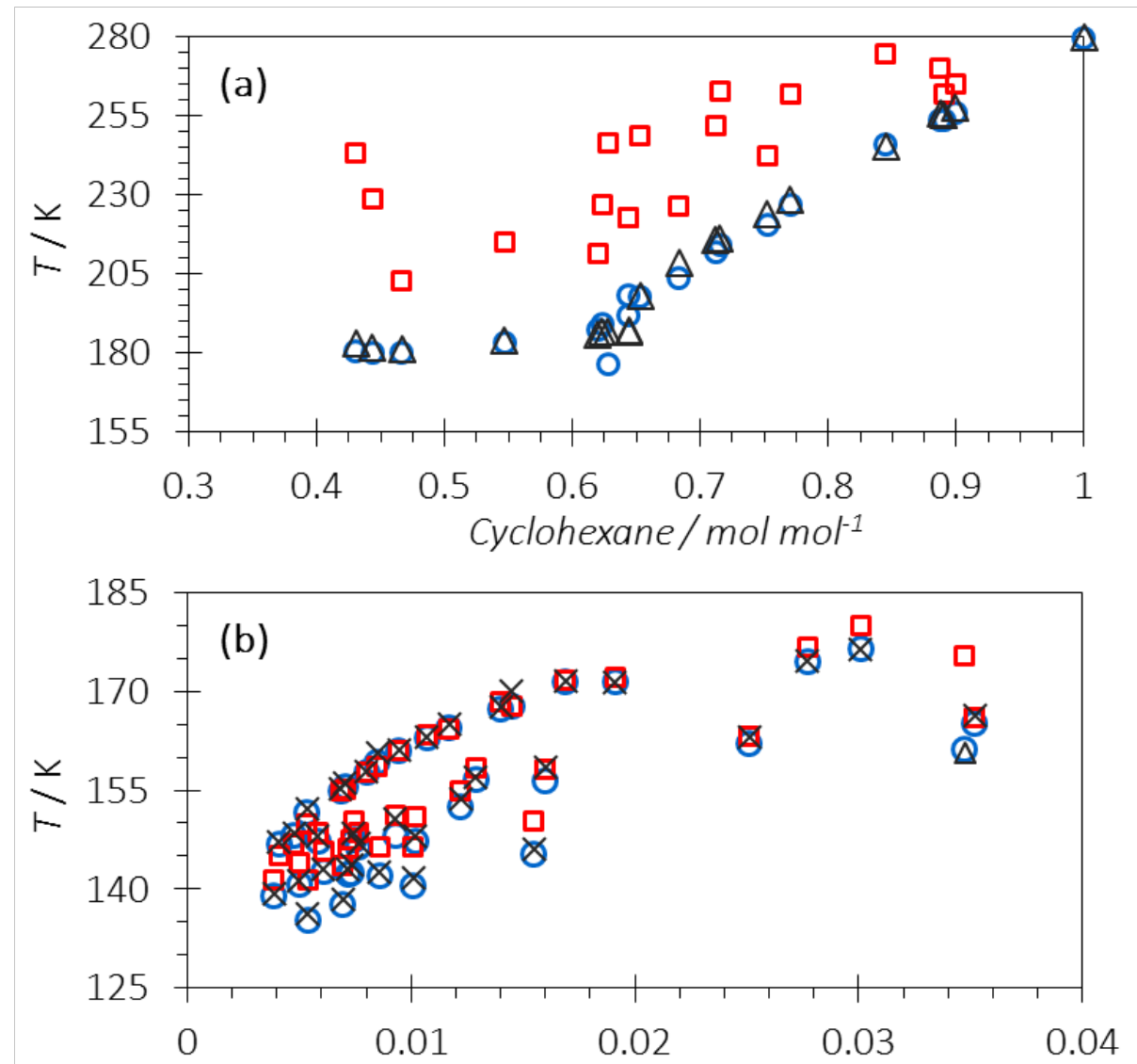

Cyclohexane $/ \mathrm{mol} \mathrm{mol}^{-1}$

OThermoFAST (0.1 to 4) MPa $\quad$ KKLSSP

$\triangle$ GPA RR-215 (2014) $\quad$ XTiffin et al. (1979)

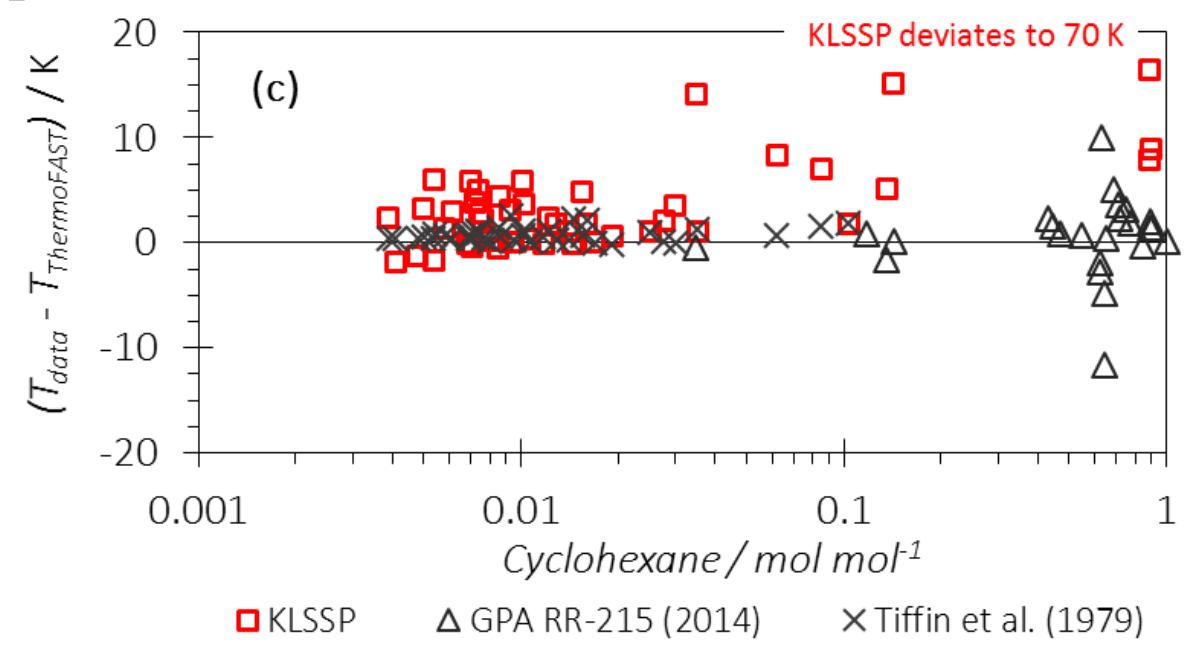

Figure 10: Solid-fluid equilibrium data for a range of multicomponent systems containing (a) low and (b) high concentrations of cyclohexane as the solute and the corresponding predictions of ThermoFAST and KLSSP. The data points were measured at different solvent compositions and pressures which accounts for the variability in the trends with concentration. The "ThermoFAST (Bubble Point)" values were calculated by specifying the solid-fluid equilibrium condition occurred on the bubble point curve, which reflects an experimental method often used for such measurements. (c) Deviations of melting temperatures predicted by ThermoFAST and KLSSP from all the experimental data [14, 23]. For clarity, only some of the data points represented in (c) are shown in (a) and (b). 


\section{Solid Phase Transitions in Mixtures}

The Peng Robinson EOS together with flash algorithms for calculating phase equilibria over wide ranges of temperature, pressure and composition, allows ThermoFAST to determine the location and nature of multiple transitions involving the appearance or disappearance of solid phases along a given pathway. The solids search algorithm presented in allows the user to search along a constant pressure pathway for all the temperatures at which a transition occurs where a solid is or becomes present upon further cooling. Often, there is only one such temperature as shown in Figure 11 for pure $\mathrm{CO}_{2}$, which shows examples of a liquid to solid transition (\#1) and a vapour to solid transition (\#2). However, in mixtures the extra degrees of freedom allow more possibilities as shown in Figure 12 for $\mathrm{CH}_{4}$ $+\mathrm{CO}_{2}$ at $5 \mathrm{MPa}$.

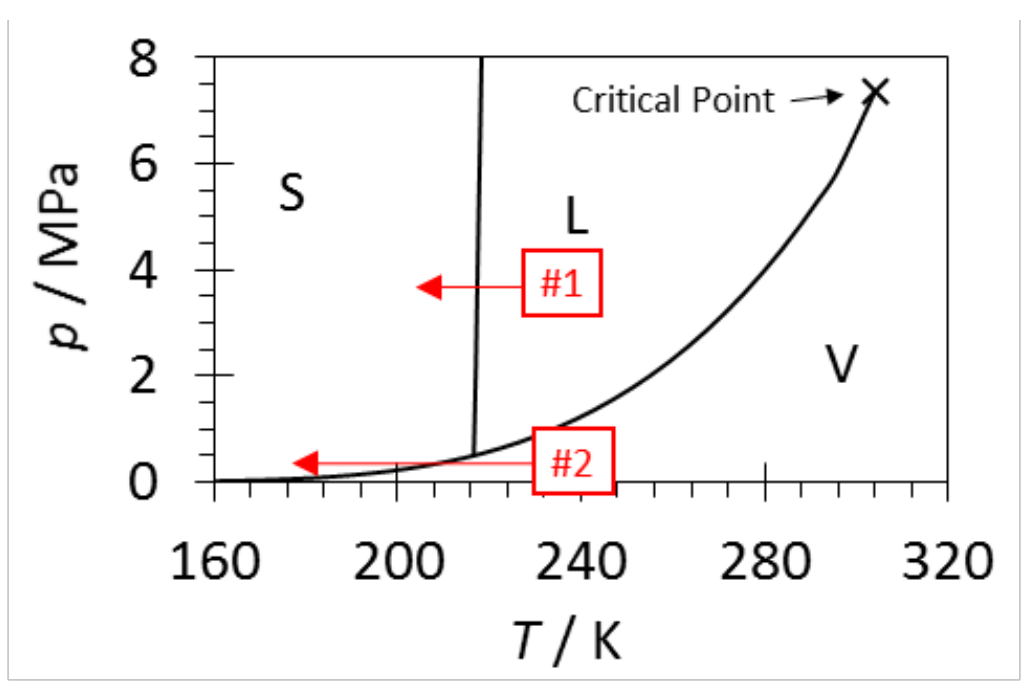

Figure 11: Pressure-temperature phase diagram for pure carbon dioxide, where $V=$ Vapour, $L=$ Liquid, $\mathrm{S}=$ Solid, and the number, \#, in the red box refers to the transition given in Table 4. 


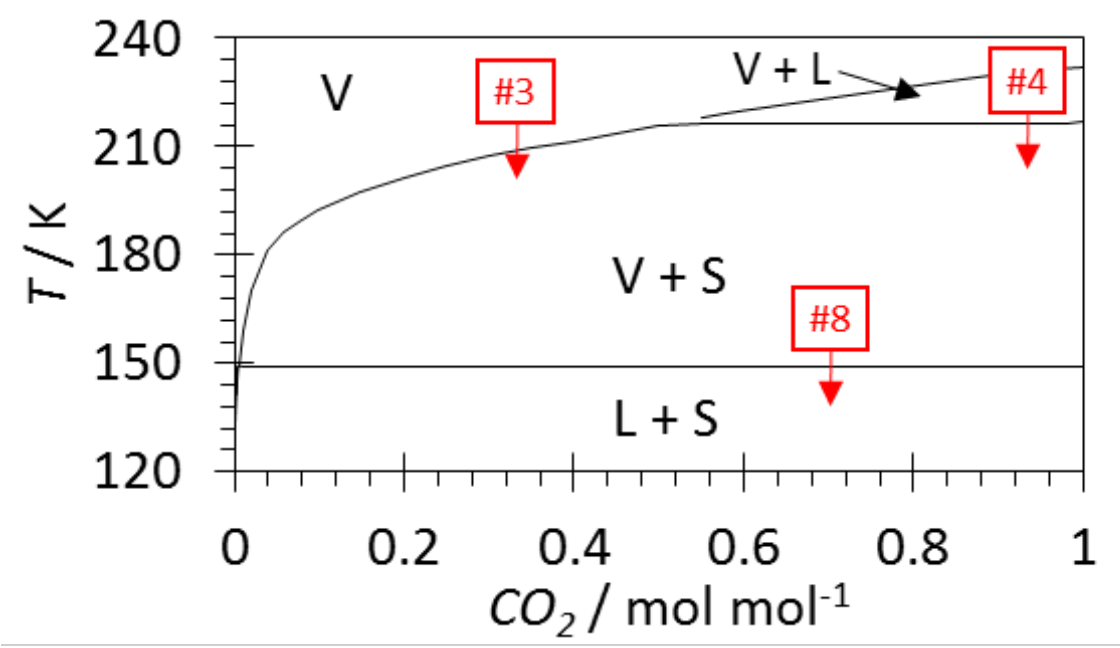

Figure 12: Temperature-composition phase diagram for the system methane and carbon dioxide $\left(\mathrm{CO}_{2}\right)$ at $5 \mathrm{MPa}$, where $\mathrm{V}=$ vapour, $\mathrm{L}=\mathrm{Liquid}, \mathrm{S}=$ Solid and the number, $\#$, in the red box refers to the transition number as given in Table 4.

For example, if temperature is reduced along a pathway of constant pressure (5 MPa) and composition $\mathrm{z}_{\mathrm{CO} 2}=0.2$ a solid phase will first appear as part of a vapour to vapour + solid transition (\#3) at around $200 \mathrm{~K}$ (see Figure 12). Further cooling along this path will produce a transition (\#8) where the vapour + solid equilibrium becomes a liquid + solid equilibrium.

Table 4 presents the Baker-May classification of transition pathways related to solid-fluid equilibrium that were identified using ThermoFAST. The classification also indicates whether each transition pathway can be the highest solid-fluid equilibrium temperature (HSET) and/or occur at a temperature below the HSET. Some of these transition pathways are only possible in multi-component mixtures as a consequence of Gibbs Phase rule. Correctly identifying which type of transition is associated with the appearance of a solid phase at a given condition is important in an LNG production context because processing in upstream operations can in principle produce a fluid mixture containing no solids that is initially at a condition below its HSET. 
Table 4: The Baker-May classification of transition pathways involving solid-fluid equilibrium for pure fluids and mixtures. The ability for the transition to be the highest solid-fluid equilibrium temperature (HSET) and/or occur at a temperature below HSET is also indicated.

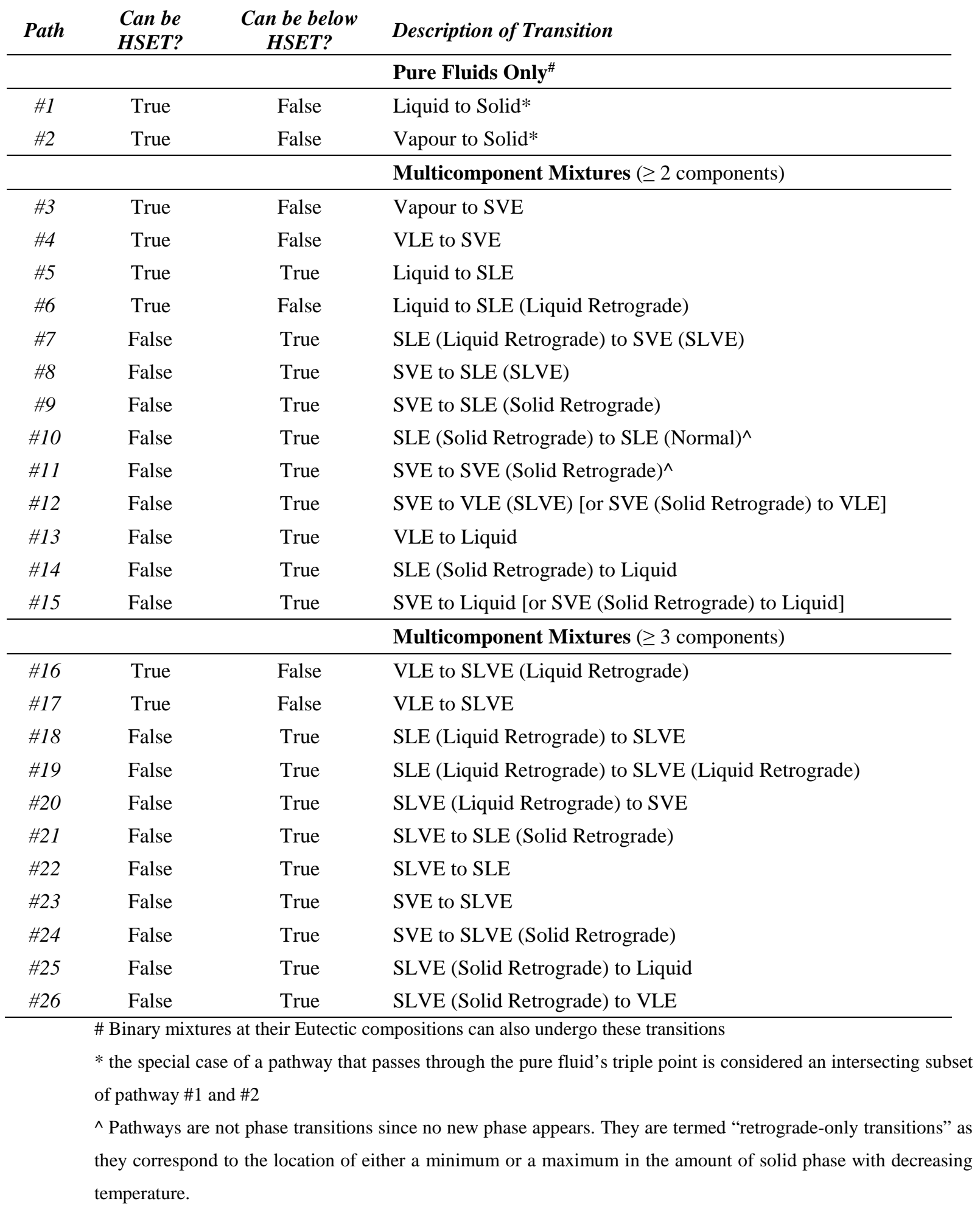




\subsection{Retrograde Solidification}

The term retrograde is used frequently in Table 4 and refers to a decrease in the amount of a given phase with decreasing temperature, in contrast to normal behaviour, where the amount of that phase would otherwise increase as the temperature is lowered. This is conceptually similar to retrograde condensation [48]. For example, the normal behaviour of a solid-liquid system is to increase the amount of solid as the temperature decreases; however, in mixtures a retrograde behaviour can be found where the amount of solid present decreases as the temperature is lowered. As a conceptual example, consider a mixture at equilibrium with vapour, liquid and solid phases initially present. Upon cooling, as components in the vapour phase begin to liquefy there could be more solvent (or liquid) to help re-dissolve the solute back into solution, thus producing retrograde behaviour of the solid.

Solid retrograde behaviour is also possible in the absence of a vapour phase. Consider the isobaric cooling of a solid-liquid system where the normal behaviour of forming additional solid would cause the remaining liquid to become excessively volatile for the system pressure. Increasing the amount of solid with cooling (i.e. normal behaviour) would then require a more extreme type of retrograde behaviour, namely the formation of a vapour phase with a reduction in temperature. Such a system would likely be unstable as the remaining, richer liquid could re-dissolve the solid, and then the heavy liquid could absorb some of the vapour phase. Rather than allow the formation of a vapour phase upon a reduction in temperature, the solid phase instead redissolves in such systems, exhibiting solid retrograde behaviour.

\subsection{Exemplar Transitions in Mixtures with Benzene}

The full search algorithm ThermoFAST utilises to locate these transitions is given in the appendices, including descriptions and examples for all of the transition pathways listed in Table 4, is available in the thesis by Baker (2018) [37]. Many of them can be illustrated via examples of binary and multi-component mixtures in which benzene is a solute. Figure 13 shows temperature composition phase diagrams for the methane + benzene binary mixture at a constant pressure of 4.0 MPa. At this pressure, the binary mixture exhibits 11 separate transition pathways, including the onset of retrograde solidification behaviour at around 200 $\mathrm{K}$ for benzene mole fractions larger than $10^{-4}(\# 9)$. At around $180 \mathrm{~K}$, this retrograde 
behaviour reverts to normal solidification as the amount of solid phase present passes through a minimum at this transition pathway (\#10).
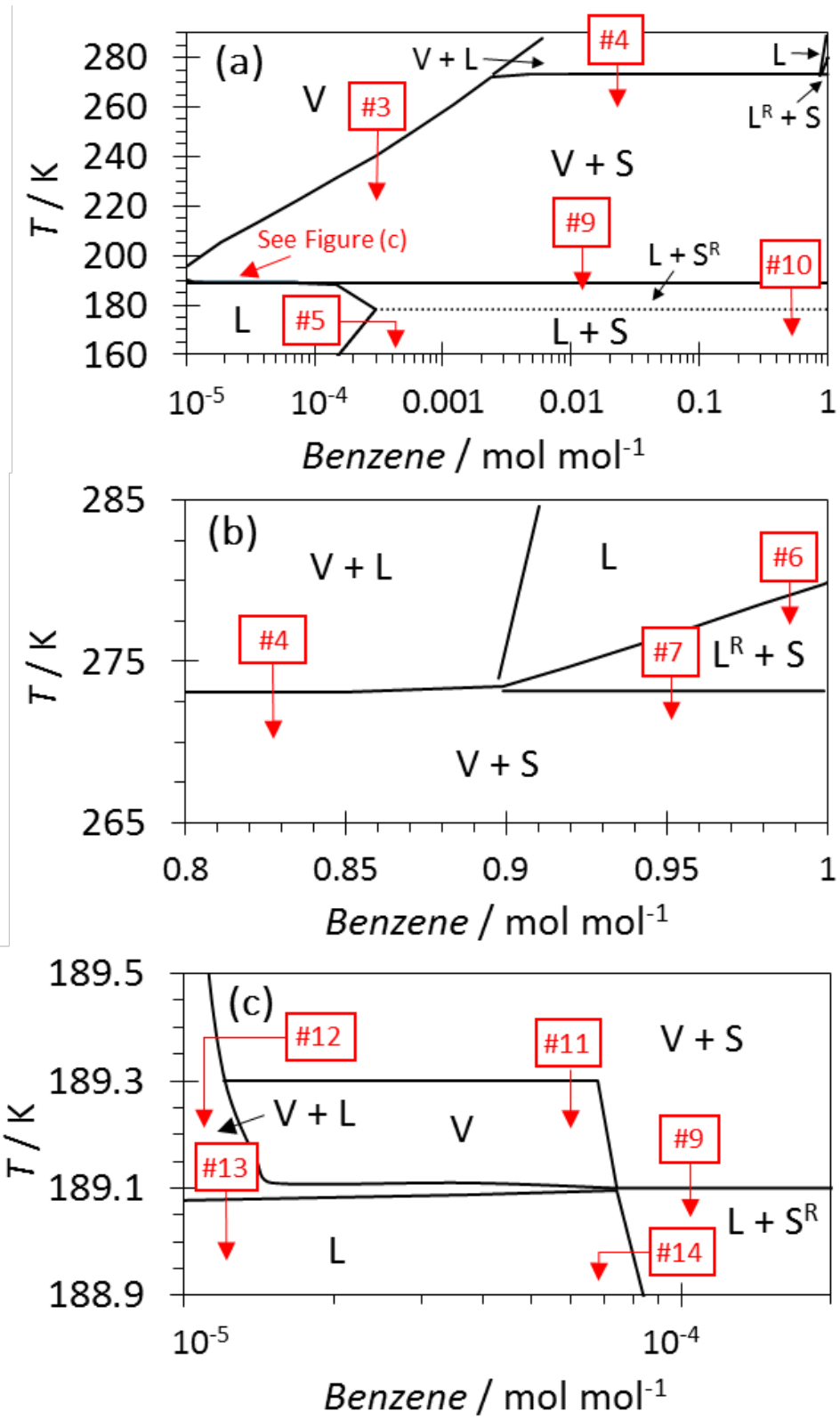

Figure 13: Temperature-composition phase diagram for the system methane and benzene at 4.0 MPa, where $V=$ vapour, $L=$ liquid, and $S=$ solid. The superscript $R$ indicates that the phase exhibits retrograde behaviour (amount decreases with decreasing temperature) ), a dashed line indicates a transition to retrograde behaviour without a phase change and the number, \#, in the red box refers to the transition number as given in Table 4. Figure (b) and Figure (c) are subsets of Figure (a).

Figure 14 shows temperature composition phase diagrams for a ternary mixture of benzene in a methane + propane solvent at a constant pressure of $5 \mathrm{MPa}$. Figure 15 shows temperature composition phase diagrams for benzene in multi-component solvent at 1.2 MPa. These systems contain further examples of unusual transition pathways, such as the onset of retrograde solidification upon cooling from a SLVE to an SLE condition (\#21), and the 
complete disappearance of a solid phase upon cooling from an SLVE to a VLE condition (\#26).
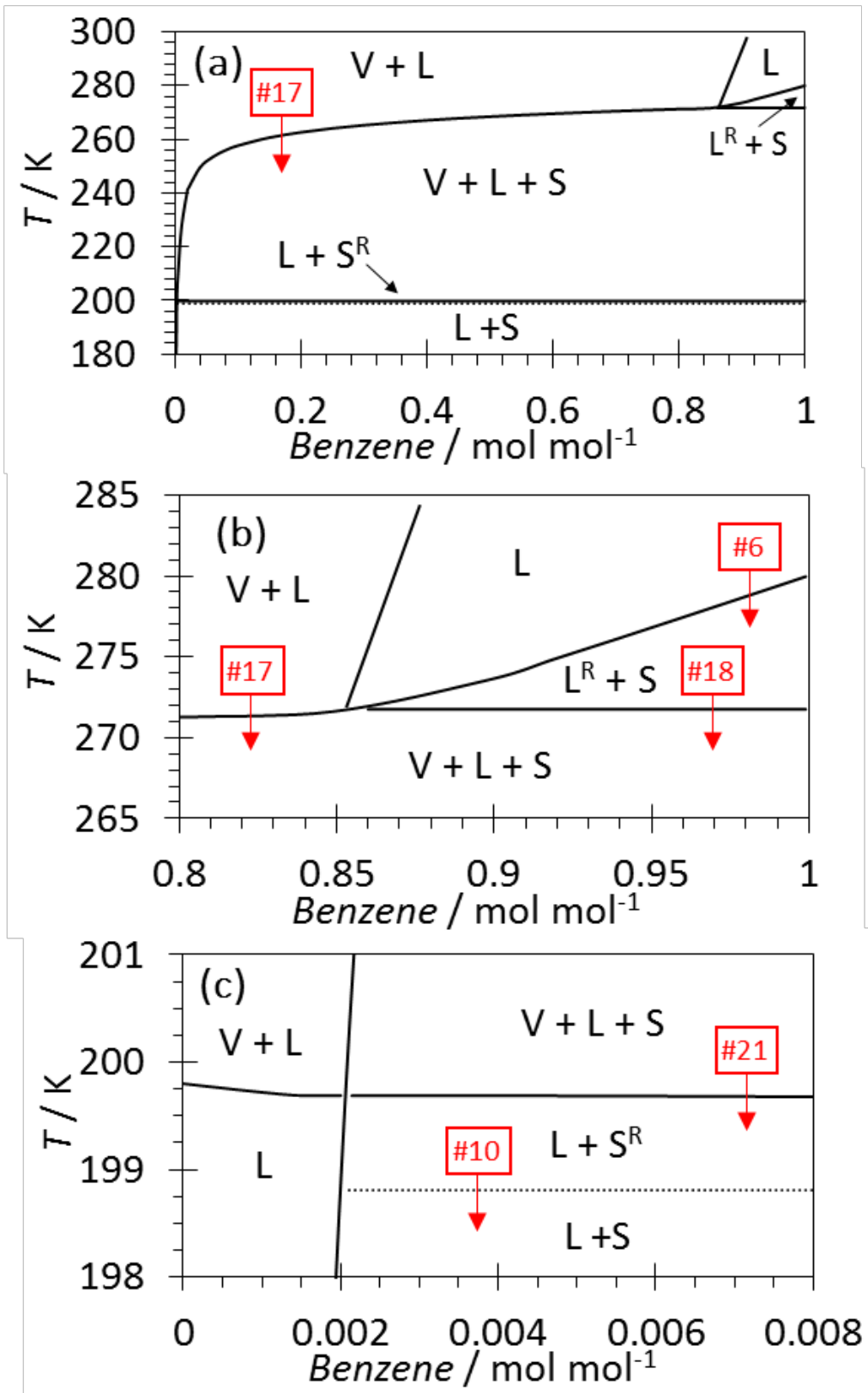

Figure 14: Temperature-composition phase diagram for the system methane, propane and benzene at $5 \mathrm{MPa}$ (excluding benzene, the solvent's composition is $0.909 \mathrm{CH}_{4}+0.091 \mathrm{C}_{3} \mathrm{H}_{8}$ ), where $\mathrm{V}=$ vapour, $\mathrm{L}=$ liquid, and $\mathrm{S}=$ solid. The superscript $\mathrm{R}$ indicates that the phase exhibits retrograde behaviour (amount decreases with decreasing temperature), a dashed line indicates a transition to retrograde behaviour without a phase change, and the number, \#, in the red box refers to the transition number as given in Table 4. Figure (b) and Figure (c) are subsets of Figure (a). 


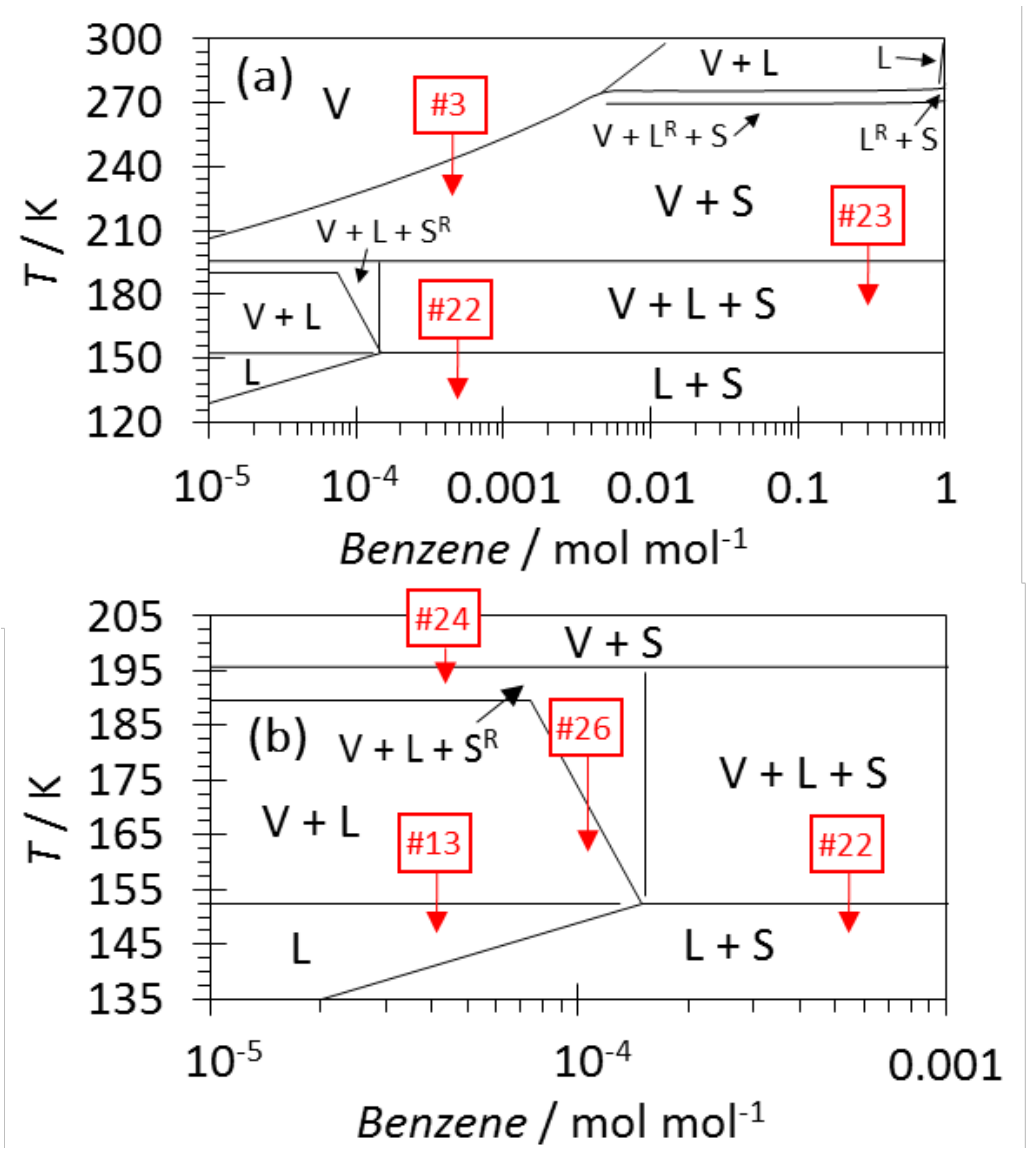

Figure 15: Temperature-composition phase diagram for the system nitrogen, methane, ethane, propane and benzene at 1.2 MPa (excluding benzene, the solvent's composition is $0.015 \mathrm{~N}_{2}+0.915$ $\mathrm{CH}_{4}+0.06 \mathrm{C}_{2} \mathrm{H}_{6}+0.01 \mathrm{C}_{3} \mathrm{H}_{8}$ ), where $\mathrm{V}=$ vapour, $\mathrm{L}=$ liquid, and $\mathrm{S}=$ solid. The superscript $\mathrm{R}$ indicates that the phase exhibits retrograde behaviour (amount decreases with decreasing temperature) and the number, \#, in the red box refers to the transition number as given in Table 4 . Figure (b) is a subset of Figure (a).

\section{Conclusion}

ThermoFAST is a new thermodynamic software package for LNG and natural gas mixtures capable of calculating solid-liquid-vapour equilibria, predicting solids formation in complex multicomponent systems, and generating full phase envelopes as well as solubility curves. It contains an improved PR cubic EOS that has been tuned to solid-fluid equilibrium data applicable to LNG and other cryogenic natural gas operations. It is recommended as a replacement to the current industry accepted tool for freeze-out predictions, the Kohn and Luks Solid Solubility Program (KLSSP). ThermoFAST is capable of considering a wider range of conditions and components than KLSSP and was tuned to represent the solid-fluid equilibrium data available for binary mixtures with an rmsd of $1.5 \mathrm{~K}$ across the entire range 
of binaries and conditions. For binaries present in KLSSP, the rmsd of the KLSSP was 19.1 $\mathrm{K}$ across the entire range of conditions, although has an rmsd of $5.6 \mathrm{~K}$ in its limited range of stated validity. For the limited solid-fluid equilibrium data available for multicomponent mixtures, ThermoFAST had a minimum and maximum rmsd of (1.0 and 2.9) K, respectively, compared to KLSSP which was (8.0 and 20.7) K, respectively.

The rapid flash algorithm's implemented within ThermoFAST allowed the exploration of phase equilibria involving solids in multi-component mixtures at conditions relevant to LNG production. The exploration revealed a set of 26 types of transition pathways related to solidfluid equilibrium that are qualitatively distinct. Many of these transition pathways exhibit the phenomenon of retrograde solidification, where the amount of solid phase present at equilibrium decreases as the temperature is lowered. To our knowledge, this prediction of retrograde solidification in hydrocarbon mixtures has not yet been verified experimentally. Future experiments should seek to verify this predicted behaviour, which if established could have useful implications for the remediation of some blockages that can form in LNG plants. 


\section{References}

1. Saadawi, H. Global Natural Gas Outlook. 2018 Ringstone Petroleum [Accessed: 08 / 02 / 2018]; Available from: https://www.spe.org/en/ogf/ogf-article-detail/?art=3762.

2. BP Energy Outlook. 2017 BP p.l.c. [Accessed: 08 / 02 / 2018]; Available from: https://www.bp.com/content/dam/bp/pdf/energy-economics/energy-outlook-2017/bpenergy-outlook-2017.pdf.

3. International Gas Union. Small Scale LNG. 2015 [Accessed: 15.05.2017]; Available from: http://www.igu.org/sites/default/files/node-page-field_file/SmallScaleLNG.pdf.

4. International Gas Union. World LNG Report. 2016 [Accessed: 15.05.2017]; Available from: www.igu.org/download/file/fid/2123.

5. Visser, A. World Fleet of LNG Carriers > 75.000 m3. 2012 [Accessed: 15.05.2017]; Available from: http://www.aukevisser.nl/supertankers/gas-SP/id703.htm.

6. Ministry of Economy Trade and Industry. Trend of the price of spot-LNG. 2017 [Accessed: 15.05.2017]; Available from: http://www.meti.go.jp/english/statistics/sho/slng/result/pdf/201704-e.pdf.

7. Madouri, A. Improvement of high heating value of commercialised liquefied natural gas of GL1Z plant by optimising the LPG extraction. in LNG-14, Doha, Qatar. 2004.

8. Kohn, J.P. and Luks, K.D., Solubility of Hydrocarbons in Cryogenic LNG and NGL Mixtures. 1976, GPA Research Report 22.

9. Kohn, J.P., Luks, K.D. and Liu, P.H., Three-phase solid-liquid-vapor equilibriums of binary-n-alkane systems (ethane-n-octane, ethane-n-decane, ethane-n-dodecane). J. Chem. Eng. Data, 1976. 21(3): p. 360-362 DOI: http://doi.org/10.1021/je60070a012.

10. Kohn, J.P., Luks, K.D., Liu, P.H. and Tiffin, D.L., Three-phase solid-liquid-vapor equilibriums of the binary hydrocarbon systems methane-n-octane and methanecyclohexane. J. Chem. Eng. Data, 1977. 22(4): p. 419-421 DOI: http://doi.org/10.1021/je60075a011.

11. Liu, P.H., Luks, K.D. and Kohn, J.P., Three-phase solid-liquid-vapor equilibria of the systems ethane-benzene, ethane-cyclohexane, and ethane-trans-decalin. J. Chem. Eng. Data, 1977. 22(2): p. 220-221 DOI: http://doi.org/10.1021/je60073a014.

12. Tiffin, D.L., Kohn, J.P. and Luks, K.D., Three-phase solid-liquid-vapor equilibriums of binary ethylene-n-alkane systems (ethylene-n-octane, ethylene-n-decane, ethylenen-dodecane). J. Chem. Eng. Data, 1978. 23(3): p. 207-209 DOI: https://doi.org/10.1021/je60078a013.

13. Tiffin, D.L., Kohn, J.P. and Luks, K.D., Solid hydrocarbon solubility in liquid methane-ethane mixtures along three-phase solid-liquid-vapor loci. J. Chem. Eng. Data, 1979. 24(4): p. 306-310 DOI: http://doi.org/10.1021/je60083a013.

14. Tiffin, D.L., Kohn, J.P. and Luks, K.D., Three-phase solid-liquid-vapor equilibriums of the systems ethylene-cyclohexane, ethylene-trans-decalin, ethylene-benzene, and ethylene-2-methylnaphthalene. J. Chem. Eng. Data, 1979. 24(2): p. 96-98 DOI: http://doi.org/10.1021/je60081a019.

15. Tiffin, D.L., Kohn, J.P. and Luks, K.D., Three phase solid-liquid-vapor equilibriums of the binary hydrocarbon systems ethane-2-methylnaphthalene, ethane-naphthalene, propane-n-decane, and propane-n-dodecane. J. Chem. Eng. Data, 1979. 24(2): p. 98100 DOI: http://doi.org/10.1021/je60081a020.

16. Chen, W.-L., Callahan, S.F., Luks, K.D. and Kohn, J.P., Effect of the presence of nitrogen on solid solubility of normal paraffins in liquid methane. J. Chem. Eng. Data, 1981. 26(2): p. 166-168 DOI: http://doi.org/10.1021/je00024a022. 
17. Chen, W.-L., Luks, K.D. and Kohn, J.P., Three-phase solid-liquid-vapor equilibriums of the binary hydrocarbon systems propane-benzene, propane-cyclohexane, butanebenzene, butane-cyclohexane, butane-n-decane, and butane-n-dodecane. J. Chem. Eng. Data, 1981. 26(3): p. 310-312 DOI: http://doi.org/10.1021/je00025a027.

18. Hottovy, J.D., Kohn, J.P. and Luks, K.D., Partial miscibility behavior of the methaneethane-n-octane system. J. Chem. Eng. Data, 1981. 26(2): p. 135-137 DOI: http://doi.org/10.1021/je00024a009.

19. Luks, K.D., Hottovy, J.D. and Kohn, J.P., Three-phase solid-liquid-vapor equilibriums in the binary hydrocarbon systems methane-n-hexane and methanebenzene. J. Chem. Eng. Data, 1981. 26(4): p. 402-403 DOI: http://doi.org/10.1021/je00026a016.

20. Hottovy, J.D., Kohn, J.P. and Luks, K.D., Partial miscibility behavior of the ternary systems methane-propane-n-octane, methane-n-butane-n-octane, and methane-carbon dioxide-n-octane. J. Chem. Eng. Data, 1982. 27(3): p. 298-302 DOI: http://doi.org/10.1021/je00029a020.

21. Luks, K.D., Kohn, J.P., Liu, P.H. and Kulkarni, A.A., Solubility of hydrocarbons in cryogenic NGL and LNG. GPA RR-033, 1975.

22. Kohn, J.P. and Luks, K.D., Solubility of Hydrocarbons in Cryogenic LNG and NGL Mixtures. GPA RR-022, 1976.

23. Jasperson, L.V., Mcdougal, R.J. and Wilson, G.M., Equilibrium Data (SLE and VLE) for Heavy and Light Hydrocarbons at Cryogenic Temperatures. Wiltec Research Co., Inc., Provo, Utah, GPA RR-215 Project 035, 2014.

24. Thol, M., Richter, M., May, E.F., Lemmon, E.W. and Span, R., EOS-LNG: A Fundamental Equation of State for the Calculation of the Thermodynamic Properties of Liquefied Natural Gases. Submitted to Fuel, 2019.

25. Kunz, O. and Wagner, W., The GERG-2008 Wide-Range Equation of State for Natural Gases and Other Mixtures: An Expansion of GERG-2004. J. Chem. Eng. Data, 2012. 57(11): p. 3032-3091 DOI: 10.1021/je300655b.

26. Rowland, D., Hughes, T.J. and May, E.F., Extending the GERG-2008 equation of state: Improved departure function and interaction parameters for (methane + butane). J. Chem. Thermodyn., 2016. 97: p. 206-213 DOI: https://doi.org/10.1016/j.jct.2016.01.005.

27. Gupta, A.K., Bishnoi, R.P. and Kalogerakis, N., A method for the simultaneous phase equilibria and stability calculations for multiphase reacting and non-reacting systems. Fluid Phase Equilib., 1991. 63(1): p. 65-89 DOI: https://doi.org/10.1016/0378-3812(91)80021-M.

28. Ballard, A.L.S., E.D., The next generation of hydrate prediction: Part III. Gibbs energy minimisation formalism. Fluid Phase Equilib., 2004. 218(1): p. 15-31.

29. Peng, D.-Y. and Robinson, D.B., A New Two-Constant Equation of State. Ind. Eng. Chem., 1976. 15(1): p. 59-64.

30. Hildebrand, J.H. and Scott, R.L., The Solubility of Nonelectrolytes. 1950, New York: Reinhold Pub. Corp.

31. Prausnitz, J.M., Lichtenthaler, R.N. and De Azevedo, E.G., Molecular Thermodynamics of Fluid-Phase Equilibria. 1998: Pearson Education.

32. Brown, T.S., Niesen, V.G., Erickson, D.D. and Inc., C., The Effects of Light Ends and High Pressure on Paraffin Formation. Soc. Pet. Eng. J., 1994. 28505.

33. Lira-Galeana, C., Firoozabadi, A. and Prausnitz, J.M., Thermodynamics of wax precipitation in petroleum mixtures. AIChE J., 1996. 42(1): p. 239-248 DOI: http://doi.org/10.1002/aic.690420120. 
34. Morgan, D.L. and Kobayashi, R., Triple point corresponding states in long-chain nalkanes. Fluid Phase Equilib., 1991. 63(3): p. 317-327 DOI: https://doi.org/10.1016/0378-3812(91)80038-W.

35. Won, K.W., Thermodynamics for solid solution-liquid-vapor equilibria: wax phase formation from heavy hydrocarbon mixtures. Fluid Phase Equilib., 1986. 30(Supplement C): p. 265-279 DOI: https://doi.org/10.1016/0378-3812(86)80061-9.

36. Assael, M.J., Trusler, J.M. and Tsolakis, T.F., Thermophysical Properties of Fluids: An Introduction to Their Prediction. 1996.

37. Baker, C.J., Phase Equilibrium Measurements and Advanced Modelling for Optimising Liquefied Natural Gas Production. PhD Thesis, The University of Western Australia, 2018.

38. Baker, C.J., Oakley, J.H., Rowland, D., Hughes, T.J., Aman, Z.M. and May, E.F., Rapid Simulation of Solid Deposition in Cryogenic Heat Exchangers To Improve Risk Management in Liquefied Natural Gas Production. Energy Fuels, 2018(32): p. 255267 DOI: http://doi.org/10.1021/acs.energyfuels.7b03057.

39. Kuebler, G.P. and Mckinley, C., Solubility of solid benzene, toluene, $n$-hexane and nheptane in liquid methane. Adv. Cryog. Eng., 1974. 9: p. 320.

40. Rijkers, M.P.W.M., Hathie, M., Peters, C.J. and De Swaan Arons, J., Experimental determination of the phase behavior of binary mixtures of methane + benzene: Part II. The solubility of liquid benzene in supercritical methane. Fluid Phase Equilib., 1992. 77(Supplement C): p. 343-353 DOI: http://doi.org/10.1016/03783812(92)85113-M.

41. Parks, G.S., Huffman, H.M. and Thomas, S.B., Thermal data on organic compounds. VI. The heat capacities, entropies and free energies of some saturated, non-benzenoid hydrocarbons. J. Am. Chem. Soc., 2013. 52: p. 1032-1041.

42. Goates, J.R., Ott, J.B., Moellmer, J.F. and Farrell, D.W., (Solid + liquid) phase equilibria in n-hexane + cyclohexane and benzene + p-xylene. The Journal of Chemical Thermodynamics, 1979. 11(7): p. 709-711 DOI: https://doi.org/10.1016/0021-9614(79)90038-7.

43. Preston, G.T., Funk, E.W. and Prausnitz, J.M., Solubilities of hydrocarbons and carbon dioxide in liquid methane and in liquid argon. J. Phys. Chem. B, 1971. 75(15): p. 2345-2352 DOI: http://doi.org/10.1021/j100684a020.

44. Pikaar, M.J., A study of phase equilibria in hydrocarbon- $\mathrm{CO}_{2}$ systems Ph.D. Thesis, Imperial College London 1959.

45. Zhang, J., Sun, J., Zhang, X., Zhao, Y. and Zhang, S., The recent development of CO2 fixation and conversion by ionic liquid. Greenh Gases, 2011. 1(2): p. 142-159 DOI: http://doi.org/10.1002/ghg.13.

46. Shen, W., He, Y., Zhang, S., Li, J. and Fan, W., Yeast-Based Microporous Carbon Materials for Carbon Dioxide Capture. ChemSusChem, 2012. 5(7): p. 1274-1279 DOI: http://doi.org/10.1002/cssc.201100735.

47. Le, T.T. and Trebble, M.A., Measurement of Carbon Dioxide Freezing in Mixtures of Methane, Ethane, and Nitrogen in the Solid-Vapor Equilibrium Region. J. Chem. Eng. Data, 2007. 52(3): p. 683-686 DOI: http://doi.org/10.1021/je060194j.

48. Katz, D.L. and Kurata, F., Retrograde Condensation. Ind. Eng. Chem., 1940. 32(6): p. 817-827 DOI: http://doi.org/10.1021/ie50366a018. 